\title{
Expression of cysteine proteinases and cystatins in parasites and use of cysteine proteinase inhibitors in parasitic diseases. Part III: Protozoa (2): Plasmodium spp.
}

\author{
Sherif M Abaza
}

Medical Parasitology Department, Faculty of Medicine, Suez Canal Uinversity, Ismailia, Egypt

\begin{abstract}
Genomic analysis of P. falciparum revealed more than thirty cysteine proteases (CPs). However, the most studied CPs are four falcipains (FPs), three dipeptidyl peptidases, a calpain and a metacaspase. Beside the main function of hemoglobin degradation, CPs are not only essential for protein trafficking, but they are also involved in egress cascade, i.e. rupture of infected erythrocytes as well as de novo RBCs invasion. In addition, studies showed their essential role in exo-erythrocytic hepatic stages, as well as oocyst production and gamete egress in mosquitoes. Accordingly, CPs inhibitors (CPIs) are of great interest in development of novel anti-malarial drugs as well as a new strategy to eliminate malaria transmission. Several compounds were investigated as CPIs including herbal extracts, known proteases with reported inhibitory potency against papain-like family, chemical compounds and synthesized derivatives as well as commercially available drugs approved for human use for other diseases. However, no commercial drug-targeting FPs has been developed yet. On the other hand, endogenous parasites cystatins (CYSs) regulate CPs and prevent inappropriate effects of host enzymes. The present review will discuss the role of essential plasmodial CPs and the importance of search for or development of potent specific selective CPI as a novel anti-marital drug. Hopefully the rapid development of highly efficient technology promises advances of expression systems using genetic tools for metabolic regulation of protein expression. This is in addition to recent technology for advanced screening directed with molecular modeling using three-dimensional construction of the target CP.
\end{abstract}

Keywords: drug targets, falcipains, malaria, plasmodial proteinases.

Received: 7 July, 2019, Accepted: 20 August, 2019.

Corresponding Author: Tel.: +20 1005243428, E-mail: smabaza@hotmail.com.

Print ISSN: 1687-7942, Online ISSN: 2090-2646, Vol. 12, No. 2, August, 2019.

Abbreviations: ADMET: Computational tool to evaluate drug absorption, distribution, metabolism, excretion and toxicity; CALP: Calpain; CP: Cysteine proteinase; CPI: Cysteine proteinase inhibitor; CSP: Circumsporozoite protein; CYS: Cystatin; DPAP: Dipeptidyl aminopeptidase; E-64: A broad spectrum CPI; FP: Falcipain; HTS: High throughput screening; MCA: Metacaspase; MSP: Merozoite surface protein; PV: Parasitophorus vacuole; SAR: Structure activity relationship; SERA: Serine-repeat antigen; VP: Vivapain; VS: Virtual screening.

\section{CONTENTS}

[I] Cysteine proteinases (CPs)

A] Falcipains (FPs)

1. FP-1

2. FP-2

3. FP-3

4. FP motifs

5. FPs and egress cascade

6. Homologs and orthologs

7. Expression system

B] Dipeptidyl aminopeptidases (DPAPs)

C] Calpain (CALP)

D] Serine repeat antigens (SERAs)

E] Metacaspases (MCAs)

F] Ovarian tumor unit-like CP (OTU)

[II] Cysteine proteinase inhibitors (CPIs)

A] Peptide inhibitors

1. Protease inhibitors

a) Non-specific protease inhibitors

b) Specific DPAP inhibitors
Page

73

81 c) Specific CALP inhibitors

d) Cysteine and serine protease inhibitors

e) Cysteine and aspartic protease inhibitors

2. Pure and synthesized compounds

a) Sole inhibitors for FPs

b) Dual actions

3. Screening technology

a) Sole inhibitors for FPs

b) Inhibitors for FPs and other enzymes

B] Non-peptide inhibitors

1. Sole inhibitors for FPs

2. Dual action

C] Peptidomimetic inhibitors

D] Allosteric site inhibitors

E] Herbal inhibitors

1. Sole inhibitors for FPs

2. Dual action

[III] Cystatins (CYSs)

CONCLUDING REMARKS

REFERENCES
86

87 


\section{[I] Cysteine proteinases (CPs)}

In three review articles published by Rosenthal ${ }^{[1-3]}$, the important roles of malarial proteases in the erythrocytic life cycle stages were designated. These stages account for malarial clinical manifestations, passing from merozoites invasion to mature schizonts, rupture of infected RBCs and release of numerous invasive merozoites. The reviewer discussed all types of proteases, including CPs, required for hemoglobin degradation in the trophozoite stag and for synthesis in subsequent stages, as well as their roles in rupture and subsequent reinvasion of new RBCs. He also claimed that knockout gene encoding falcipain 2 (FP-2) led to a transient block in hemoglobin breakdown with significant increase in parasite sensitivity to CPIs. In contrast, disruption of the gene encoding FP-1 showed its essential role for oocysts production in mosquitoes. Other than FPs, $P$. falciparum genome revealed possession of three dipeptidyl aminopeptidases (DPAPs), previously recognized as calpain (CALP) homologs, and three serine-repeat antigens (SERAs) that have a cysteine motif. As it was only localized in the food vacuole, the reviewer discussed the role of DPAP1 in hemoglobin breakdown, in addition to the roles of DPAP- 3 and SERA- 5 in egress cascade. Accordingly, he suggested FP-2, DPAPs 1 and 3, and SERA- 5 as potential anti-malarial drug targets ${ }^{[3]}$.

In an attempt to characterize putative proteases in P. falciparum, a group of American investigators ${ }^{[4]}$ predicted 92 proteinases using comparative genomic analysis. Their prediction was confirmed by further phylogenetic analysis. Among them, 88 proteases were identified with their assigned transcribed proteins using microarray analysis, and reverse transcript PCR. The transcript proteinases were classified and the highest (36\%) were found to belong to CPs. Beside the identified and characterized CPs at that time, the investigators identified only two new potentially essential CPs; CALP and metacaspase (MCA). The first is a calcium-activated CP and was suggested as an essential catalytic enzyme in merozoite invasion. Its usefulness as anti-malarial drug target was suggested for two reasons. One, was the discovery of a typical endogenous CALP substrate (protein kinase C, PKC) in P. falciparum genome that is crucial for signal transduction pathways affecting biology and host-parasite interactions. Two, it is not similar to host CALPs, therefore use of inhibitors would have minimal effect on the host. Although previous studies neither reported apoptosis in $P$. falciparum, nor presence of MCA in protozoa, the investigators recommended further studies to investigate the role of plasmodial MCA as potential anti-malarial drug target ${ }^{[4]}$.

In 2011, a fourth review article was published and the reviewer tabulated the stages of gene expression of CPs in P. falciparum and P. vivax using two methods; immunoblotting and proteomic screening ${ }^{[5]}$. The trophozoite is the only stage that showed gene expression of all FPs and vivapains (VPs) which are the FP homologs in P. vivax. Gene expression of FP-1 and VP-4 was detected in ring and schizont stages Added to the previous two CPs, FP-3 was also detected in schizont stage. While FP-1 gene expression was detected only in sporozoites, VP-4 was only detected by immunoblotting in gametocytes. The reviewer suggested that the transient accumulation of undegraded hemoglobin in the food vacuole in response to knockout gene encoding FP-2 was compensated with FP-3 gene expression in late trophozoite $(12 \mathrm{~h}$ later after FP-2 expression). For $P$. vivax, the reviewer attributed the fewer studies working on $P$. vivax CPs to two reasons; unavailability of continuous in vitro $P$. vivax cultures and limited animal models (only primates). The reviewer observed that FPs and VPs have a specific unique conserved role of their motif and time of expression to facilitate their main function. In other words, they might have developed gradually to efficiently degrade host hemoglobin. Accordingly, he recommended further studies to design potent inhibitors for FPs and VPs assisted by three-dimensional structure guided technology ${ }^{[5]}$.

Beside the above mentioned functions, Plasmodium CPs proved to catalyze circumsporozoite protein (CSP), the major surface protein of Plasmodium sporozoites. It was found that CSP facilitates sporozoite adhesion to the host hepatocytes and subsequent invasion, and it should be cleaved prior to adhesion. A group of American scientists succeeded to identify its cleavage site by plasmodial $\mathrm{CPs}^{\left[{ }^{[6]}\right.}$. Using pulse-chase metabolic labeling experiments in absence and presence of different protease inhibitors, they found that more than $80 \%$ of the labeled CSP was cleaved after $2 \mathrm{~h}$ in absence of inhibitors. In contrast, E-64, a well-known broad spectrum CPI, inhibited CSP cleavage and subsequently inhibited both in vitro and in vivo sporozoites ability to adhere and invade their target cells. Using inhibitors of serine, metallo- or aspartyl-proteases were not effective to inhibit CSP functions ${ }^{[6]}$.

\section{A] Falcipains (FPs)}

1. FP-1: Rosenthal and Nelson ${ }^{[7]}$ identified the first $\mathrm{CP}$ in the trophozoite of $P$. falciparum that has an essential role as potent hemoglobinase. The American investigators named the $28-\mathrm{kDa}$ protein "trophozoite CP", termed latter as falcipain-1 (FP-1). They succeeded to synthesize its encoding gene and primed it with $P$. falciparum genomic DNA using PCR. Although FP-1 is expressed by trophozoites, the investigators did not observe its essentiality for development of erythrocytic stages. This was confirmed by two American studies ${ }^{[8,9]}$. In the first study, the investigators succeeded to identify gene expression of all $P$. falciparum FPs in malarial stages of gametocytogenesis. Results revealed upregulation of FP-1 transcripts in gametocytes. Knockout gene encoding FP-1 neither affected the morphological features of gametocytes or gametes, nor parasite sensitivity to CPIs. However, it significantly reduced oocyst production when fed to a mosquito. Accordingly, the investigators concluded the potential use of FP-1 as new strategy in malaria transmission ${ }^{[8]}$. 
Meanwhile, CPIs did not inhibit erythrocyte invasion by merozoites in both wild type and knockout parasites ${ }^{[9]}$. It was found that FP- 1 shares only $\sim 40 \%$ of sequence identity to the other FPs. In addition, it was detected in the transcriptome and proteomes of Plasmodium asexual and sexual erythrocytic stages ${ }^{[10]}$ as well as in sporozoites $^{[11]}$.

2. FP-2: In 1995, the second trophozoite 'P. falciparum CP' (FP-2) was recognized in hemoglobin degradation in an acidic environment of trophozoite food vacuole ${ }^{[12]}$. It is well known that hemoglobin degradation into heme and globin is an essential process in Plasmodium life cycle, as heme is processed into malarial pigment, and globin is hydrolyzed to free amino acids essential for parasitic stages synthesis. To characterize the catalytic role of $P$. falciparum FP-2 in the initial step for globulin breakdown, Salas et al. ${ }^{[12]}$ succeeded to express its functional proteolytic activity, i.e. as a recombinant form, in globulin hydrolysis of denatured and native hemoglobin. Results revealed that FP-2 is responsible for multiple cleavages resulting in small peptides shown in SDS-PAGE gel. Moreover, quantitative assays showed that FP-2 was capable of degrading hemoglobin at the rate of $1-3 \mathrm{pg} /$ parasite/h, i.e. $25-75 \%$ of $\mathrm{RBC}$ hemoglobin/parasite/h. Another American group of investigators demonstrated that, similar to all CPs, FP-2 required activation prior to its proteolytic activity. They also observed high transcriptomics of the gene encoding FP-2 in the early intra-erythrocytic stages ${ }^{[13]}$. It was in the year 2000 when FP-2 was purified using affinity chromatography. Its amino acids sequence as well as that of its encoding gene were identified. It was observed that FP-2 constituted $\sim 93 \%$ of trophozoite $\mathrm{CP}$ activity, and has interesting features compared with other papain family CPs. Among these features is the possession of a large prodomain, a feature observed in all other proteases (aspartic and metalo-proteases) that contribute with FP-2 in hemoglobin degradation. In their immunofluorescence studies, pro-FP-2 was detected in parasite cytosol as reservoir, and its detection was transit in food vacuole to be immediately activated for hemoglobin degradation ${ }^{[14]}$.

During the period 2004-2009, American investigators published three studies. In the first, they observed that FP-2 has a unique feature different from other previous studied papains; i.e. it does not require its prodomain for folding to an active $\mathrm{CP}$. Instead its folding was mediated by amino acids at its $C$ terminal extension of its mature enzyme ${ }^{[15]}$. One year later, the second study demonstrated that FP-2 possesses a non-ordinary motif (14 amino acids) near the $C$ terminal of its catalytic domain. Removal of this motif didn't affect FP-2 ability to cleave other protein substrates, but only failed to hydrolyze hemoglobin. Therefore, the investigators concluded that this motif has double functions; binding with hemoglobin prior to degradation, and subsequent regulatory inhibition by its prodomain ${ }^{[16]}$. As FP-2 inhibition is regulated or mediated by conserved amino acid residues in its prodomain, the third study succeeded to identify 61 amino acids. Interestingly, only the $11 \mathrm{kDa}$-terminal region of FP-2 prodomain was found essential for efficient FP-2 regulation and inhibition. In addition, structure-function studies demonstrated that only a small portion of the prodomain C terminal (leucine ${ }^{155}$ asparagine $^{216}$ ) was sufficient for inhibition of the mature FP-2 ${ }^{[17]}$. Three other studies reported similar results $^{[18-20]}$, and accordingly, further studies to develop new anti-malarial drugs based on FP-2 motif of its C terminal were recommended.

Indian investigators demonstrated that FP-2 was transferred from cytosomal vesicles to the food vacuoles using its $\mathrm{N}$ terminal region. They succeeded to identify amino acids sequence of the $\mathrm{N}$ terminal region, and recommended further studies to use it as an anti-malarial drug target ${ }^{[21]}$. For detailed analysis of FP-2 active site, its crystal structure was determined in a complex with E-64. American investigators observed that its active site that bound with its inhibitor, as a substrate, was altered by amino acid substitutions. Based on the obtained results, the investigators concluded that determination of FP-2 crystal structure would help developing a specific inhibitor as novel anti-malarial drug ${ }^{[22]}$.

A study conducted in USA observed that some mature FP-2 knockout parasites recovered from the alterations observed using CPI. Using real time $\mathrm{PCR}$, the investigators noted increased transcription of the FP-2 copy (FP-2') over that observed in wild type parasites. On knockout of the gene encoding FP2 , the investigators observed significant increased transcription of FP-1 and FP-2' in rings and FP-3 in all stages with specific increase in trophozoites. The investigators suggested the presence of a copy of the gene encoding FP-2', located on chromosome 11 close to genes encoding FP-2 and FP-3, and that its expression takes place only to allow delayed replacement of FP-2 function ${ }^{[23]}$. Later, gene encoding FP-2 ${ }^{`}$ was isolated and its transcripts were observed at the trophozoite stage. The investigators suggested another FP-2 function during parasite egress ${ }^{[24]}$. Similar results were obtained when recombinant FP-2 was constructed and its biochemical properties were compared to those of FP2. The investigators observed similar $\mathrm{pH}$ 5.5-7.0 for its activation and their preference to the same substrate (hemoglobin) ${ }^{[25]}$.

The zymogenic form of food vacuolar FP-2 is a membrane-bound protein in Plasmodium parasitophorus vacuole (PV). To characterize its trafficking signal pathway to the food vacuole, the investigators linked FP-2 prodomain with green fluorescent protein. This led to a trafficking signal that is bipartite in nature, with one motif on either side; luminal and cytoplasmic portions. In other words, a signal targeting luminal portion to carry the zymogenic form from PV and another signal targeting cytoplasmic portion to deliver it to the food vacuole. Interestingly, presence of lysine and phenylalanine-valine motifs in the luminal and cytoplasmic portions, respectively, 
essentially contributes toward proper targeting and efficient trafficking to the food vacuole. The investigators confirmed their results using mutant FP-2 prodomains and they observed failure of trafficking at the plasma membrane ${ }^{[26]}$.

Following hemoglobin degradation, free heme (ferriprotoporphyrin IX) is released in the food vacuole inducing oxygen-derived free radical formation that causes protein and DNA oxidation. To avoid its hazardous effects, Plasmodium spp. expressed heme detoxification protein (HDP) converting heme to $\beta$-hematin, also known as hemozoin. This protein is thought to be delivered through a subsequent endocytosis of the infected $\mathrm{RBCs}^{[27]}$. Five years later, a group of American and Indian investigators identified a multi-protein complex in $P$. falciparum food vacuole. The identified complex includes two FPs (2 and 2'), two aspartyl proteinases (plasmepsins II and IV), histoaspartic protease and HDP. Using immunoelectron microscopy and mass spectrophotometric analysis, the investigators provided evidence that FP-2 and HDP contributed to the efficient conversion of heam to hemozoin ${ }^{[28]}$. Similar results were obtained when Marques and his colleagues ${ }^{[29]}$ concluded that the binding between heam and FP-2 provided a mean for detoxification and transformation to hemozoin.

On the other hand, because the mode of action of artemisinin (ART) is to prevent hemezoin formation, several studies documented that mutations in the gene encoding FP-2 was linked with ART drug resistance, especially in areas with selected ART high pressure ${ }^{[30-32]}$. Analysis of polymorphisms in genes encoding FPs 2 and 2 in ART resistant strains obtained from Southeast Asia showed significant more single nucleotide polymorphisms (SNPs) with non-synonymous mutations that were located more in the prodomain than in core enzyme ${ }^{[33]}$. Furthermore, Australian researchers suggested that $\mathrm{FP}-2$ is essential to render ring stages sensitive to ART. Results showed that FP-2 delayed expression was followed by significant ART resistance ${ }^{[34]}$.

3. FP-3: In the year 2001, FP-3 was identified and characterized and it showed $65 \%$ identity to FP-2, however, the investigators detected few differences among them. It was observed that FP-3 is activated only at acidic $\mathrm{pH}$ in food vacuole with greater stability and activity than FP-2 against native hemoglobin. The obtained results suggested that FP-3 is the principal hemoglobinase, while FP-2 acts under specific conditions such as lower acidic or neutral $\mathrm{pH}$, i.e. it is activated in extra-vacuolar compartments ${ }^{[35]}$. FP-3 appears to have an essential role in Plasmodium life cycle stages, as knockout of the FP-3 gene was lethal ${ }^{[23]}$. Later, it was found that FP-3 showed similarity to FPs 2 and 2 regarding possession of 1) a specific conserved motif ( 10 amino acids) near the $C$ terminal of its catalytic domain to bind with hemoglobin prior to degradation ${ }^{[20]}$; 2) lysine and phenylalaninevaline motifs in luminal and cytoplasmic portions of its prodomain for efficient trafficking to the food vacuole $^{[26]}$; and 3) specific residues contributed to its activity as potent hemoglobinase. Residues substitution showed activity on peptide substrates than wild type FP-3, but was not efficient as hemoglobinase ${ }^{[36]}$.

In two American studies, two important points were observed. During gametocytogenesis, FP-3 was expressed in stage $\mathrm{V}$ gametocytes to be localized in vesicles along infected RBC' surface. The investigators concluded its essential role in gamete egress ${ }^{[37]}$. Similarly, as observed in FP-2, FP-3 active site binding with its inhibitor, as a substrate, was altered by amino acid substitutions. Similarly, Kerr and his colleagues ${ }^{[22]}$ recommended future studies to determine FP-3 crystal structure for successful development of novel antimalarial drugs.

4. FP motifs: Figure (1) summarizes FPs structuralfunctional relationship ${ }^{[17]}$. There are several unique features of FPs from other papain family CPs. First, the mature domain contains highly conserved active sites residues (cysteine, histidine and asparagine) similar to all other mature CPs domains. However, the mature domain in FPs have two essential unique terminals; a C-terminal acting as a hemoglobin binding domain (red ring), between histidine and asparagine, and a $\mathrm{N}$-terminal extension acting as a refolding domain (blue ring). Second, the C-terminal in all other papain family CPs have an extension motive of 14 amino acids, while motifs in FPs are made up of a 14 residue $\beta$-hairpin specifically to bind with hemoglobin. Third, other papain family enzymes require the prodomain for proper refolding of the enzyme activity. In contrast, the refolding domain; the N-terminal extensions $(12$ amino acids) is essentially required for correct folding of the mature domain to active FP, but is not required for enzyme activity. Fourth, as conserved in papain family, FPs prodomain structure has ERFNIN and GNFD motifs. However, the FPs prodomain have additional small four domains; 1) cytosolic N-terminal with 35 amino acids, 2) a 20 amino acid transmembrane domain, 3) a 188 amino acid luminal domain and 4) the inhibitory domain in the C-terminal part. The transmembrane domain is required for entry into the endoplasmic reticulum. A stretch of 20-amino acids in the luminal portion and a 10-amino acid stretch in the cytoplasmic portion are essential for food vacuolar trafficking. A certain segment at the $\mathrm{C}$ terminal (leucine $^{155}$-asparagine ${ }^{216}$ ) with ERFNIN and GNFD motifs are essential to mediate prodomain inhibitory activity ${ }^{[17]}$. Furthermore, mutagenesis studies showed that certain residues at the interface of pro- and mature domains are responsible for formation of salt bridges and hydrophobic interactions. These "hot spot" interactions are essential for prodomain dissociation and FPs auto-processing ${ }^{[38]}$.

Pandey and Dixit ${ }^{[39]}$ in their review claimed that MEROPS website identified more than 40 members with similar $\mathrm{N}$ terminal extension, among them $18 \mathrm{CPs}$ (FPs and homologs from other plasmodial species). It 


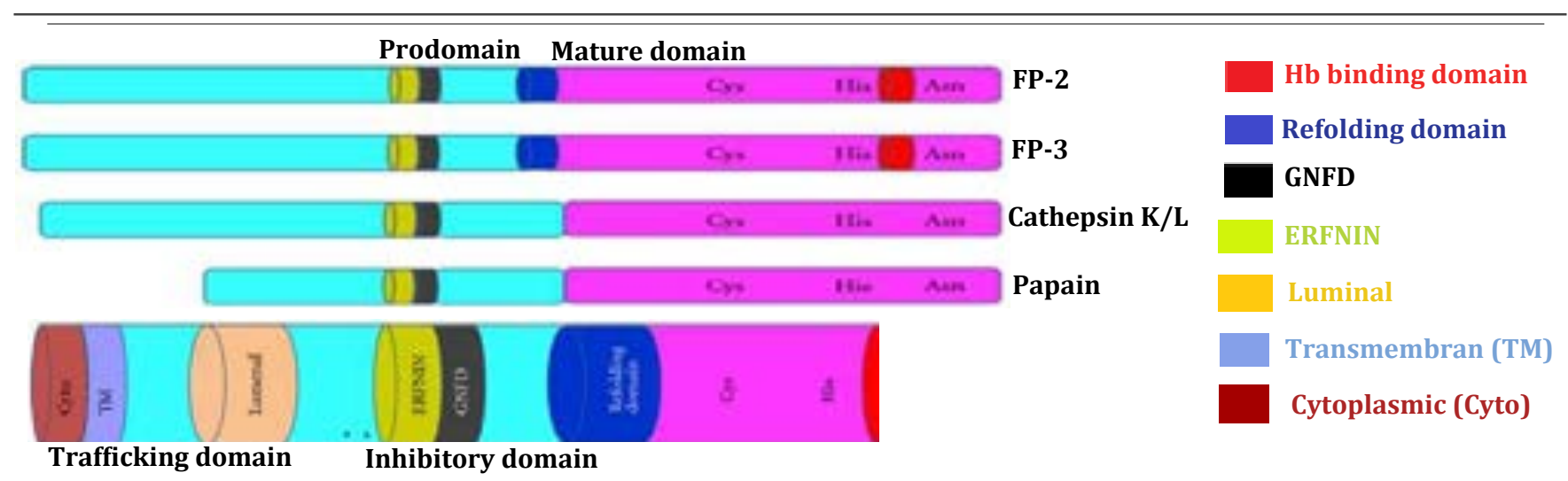

Fig. 1. Unique features of FPs. Cited from Pandey et al. ${ }^{[17]}$.

FPs 2 and 3 are synthesized as zymogens $(\sim 50 \mathrm{kDa})$. They consist of pro- and mature domains. Prodomains have their inhibitory motifs ERFNIN and GNFD covering the active site cleft of the mature domain to prevent its activation. Trafficing domain includes three subdomains; luminal, transmembrane and cytoplasmic. Zymogens are transported via the endoplasmic reticulum/Golgi network to the food vacuole, where under its acidic environment, the prodomain dissociates and releases $\sim 27 \mathrm{kDa}$ active enzyme.

was proved that a refolding domain is also essential for the stability of the mature domain of FPs. Sequence conservation among the C-terminal extension motifs of FPs (hemoglobin binding) is not as high as in their N-terminal extension (folding). An important observation indicated that FPs and other proteases contributing in hemoglobin degradation and egress and de novo RBCs invasion cascade use similar mechanisms for biological control, but use different specific means mediating interactions with their substrates and inhibitors ${ }^{[39]}$. Also in another review, this extension motif was suggested as a novel anti-malarial drug target ${ }^{[40]}$.

5. FPs and egress cascade: In the year 2000, American investigators showed that Plasmodium trophozoites and schizonts possess proteinases involved in cleavage of ankyrins that are required to maintain the integrity of the plasma membranes. This proteolytic activity is significantly inhibited by several CPIs and cystatin (CYS), but not by aspartic nor serine inhibitors. The investigators suggested that expressed CPs contribute in parasite egress and de novo RBCs invasion ${ }^{[41]}$. It was observed that FPs proteolytic activity against RBCs membrane ankyrin was significantly increased at the late trophozoite and schizont stages. Several studies showed that FP-2 contributes with FP-3 in hemoglobin degradation in early trophozoite, and is singly involved in cleavage of RBCs membrane ankyrin in late trophozoite ${ }^{[42-44]}$. In association with merozoites egress, the infected RBCs undergo morphological and physiological changes involving enlargement of PV and increased permeability with cytoskeleton destabilization. In the year 2011, Chandramohanadas et al. ${ }^{[45]}$ studied the molecular basis of both biochemical and biophysical events associated with merozoite egress from $P$. falciparum-infected RBCs. The obtained results showed that: 1) during late schizont stage, the classical spherical shape of infected RBCs is maintained by the unbroken membrane of its PV; 2) serine protease activity is the first step involved in hydrolysis of PV membrane, assisted by parasite erythrocyte proteins that bind to the cytoskeleton of infected RBCs modifying their membrane biophysical properties; 3) CPs contribute in membrane rupture leading to flattening and loss of infected RBCs shape; 4) merozoites find their way to complete a new cycle. It is worth mentioning that parasite erythrocyte proteins are well linked to increased rigidity and cytoadherence commonly associated with malignant malaria. Accordingly, the investigators concluded that development of an efficient simultaneous cysteine and serine protease inhibitor can be a potential novel antimalarial drug ${ }^{[45]}$.

The role of Plasmodium CPs proteolytic activities was also observed in sporozoite egress from Anopheles midgut. German scientists succeeded to identify SERA transcriptomic expression. They called it egress CP1 (ECP1) as it is linked to sporozoites egress from mature ruptured oocysts in Anopheles salivary glands. A $P$. berghei mutant carrying ECP1 formed viable sporozoites but they were unable to egress from mature oocysts. Viewed by phase-contrast microscopy, mutant sporozoites were arranged in circles and displayed continuous circular movement around a central axis. In contrast, wild type sporozoites were arranged in radial fashion with few bending and flexing movements preparing themselves for egress from the oocyst. In addition, mutant sporozoites were unable to cause infection even with 10 -fold higher doses than wild type sporozoites. Accordingly, the investigators suggested using ECP1 as a new strategy for vector control and to eliminate malaria transmission ${ }^{[46]}$. Using electron microscopy and different proteases inhibitors, the investigators observed that plasmodial CPs contributed with serine and aspartic proteinases in facilitating gametocytes egress from the ruptured $\mathrm{PV}^{[47]}$.

It was found that a malarial cGMP-dependent protein kinase G(PKG) activates plasmodial proteinases in egress cascade. Egress is an essential step in Plasmodium life cycle in human host, and merozoites in late schizont have to escape through the two membranes of PV and RBC. The investigators used selective inhibitors of either PKG or CPs to demonstrate 
egress steps utilizing several methods showing $P$. falciparum life cycle stages. First, PV membrane was permeabilized 10-30 min before PKG expression. Then, infected RBC underwent an abrupt, dramatic shape change as a result of sudden breakdown of its cytoskeleton. Finally, the RBC formed a single pore followed by blebbing and vesiculation to allow release of new invading merozoites ${ }^{[48]}$.

Although Plasmodium subtilisin-like proteases (SUBs) are serine proteinases, their essential role in egress cascade cannot be ignored. Plasmodium exoneme, microneme, and mononeme are characterized organelles that are identified as stores for mature SUB1, SUB2 and rhomboid-1 (ROM1), respectively. British investigators showed that: 1) While SUB1 is involved in merozoites egress, SUB2 is required for merozoites de novo RBCs invasion; 2) Degradation of PV membrane and breakdown of both RBC' membrane and cytoskeleton is essentially done by SUB1, SUB2 and ROM1 via release of several proteins; 3) SERAs 5 and 6 , and merozoite surface protein 1 (MSP1) are cleaved and activated by SUB1, while SUB2 activates MSPs 6 and 7. On the other hand, ROM1 contributes with SUB2 to catalyze the intermembrane cleavage of merozoite adhesin named apical membrane antigen (AMA1) leading to de novo RBCs invasion ${ }^{[49]}$.

6. Homologs and orthologs: Comprehensive studies of $P$. vivax CPs were limited due to inability to culture $P$. vivax. However, two studies were conducted in 2004 to clone genes encoding VPs 2 and 3, located on chromosome 9. Results revealed that VPs shared FPs in their usual characters e.g. acidic $\mathrm{pH}$ and reducing conditions for their activity, and breakdown of hemoglobin erythrocyte cytoskeletal protein 4.1; as well as unusual features such as large prodomains, short N-terminal extensions on the catalytic domain, and a C-terminal insertion ${ }^{[50.51]}$. Six years later, the investigators succeeded to amplify the full-length of the gene encoding VP-4 from $P$. vivax isolated from a Korean patient's blood. Recombinant VP-4 demonstrated dual substrate specificity depending on the surrounding micro-environmental $\mathrm{pH}$ either at acidic $\mathrm{pH}$ (5.5), or neutral pH (6.5-7.5). VP-4 was localized in the food vacuoles and cytoplasm of the erythrocytic stage of $P$. vivax. It showed maximal activity against actin at neutral $\mathrm{pH}$, against hemoglobin at acidic $\mathrm{pH}$, while that against plasmepsin-IV (an aspartyl proteinase) was at neutral or acidic $\mathrm{pH}^{[52]}$. Later, two studies showed that plasmepsin-IV acted synergistically with VPs 2 and 3 in hemoglobin degradation ${ }^{[5,53]}$.

Similarly, vinckepain-2 and berghepain-2, expressed in rodent $P$. vinckei and $P$. berghei, respectively were characterized. Both showed important differences from FP-2 as regard their substrate preference and inhibitor sensitivity and specificity ${ }^{[54]}$. Because all in vivo studies for development of a novel anti-malarial drug were conducted in murine models, trials to develop a specific inhibitor targeting FP-2 substrate in its specific binding pocket, became a challenge. Since
$P$. vinckei-infected mice failed as an animal model in high throughput screening (HTS) of inhibitors, Chan et $a{ }^{[55]}$ described the first study utilizing $P$. berghei to characterize berghepain-2. The investigators detected two important differences; a narrower range of $\mathrm{pH}$ optima, and exhibition of different substrate specificities at positions 63 and 230 amino acids that essentially contributed to their substrate specificity in its binding pocket. Later, another study succeeded in replacing 13 amino acids in berghepain- 2 residues. The mutant enzyme showed efficient activity, substrate preference and inhibitor sensitivity as well as specificity similar to FP-2 ${ }^{[56]}$. On the other hand, to well understand the role of FP-1 across Plasmodium life cycle stages, the investigators studied its ortholog, berghepain-1, in the rodent $P$. berghei. Unlike berghepain-2, its expression proved to be conserved in all species with an essential role in the infectivity capability of both hepatic and erythrocytic merozoites. However, its impact is significantly more pronounced in hepatic merozoites. In addition, berghepain-1 knockout parasites showed a pronounced delay in erythrocytic infection after localization of mutant sporozoites in the liver. Accordingly, the investigators suggested that hepatic and erythrocytic merozoites are distinct populations that differentially rely on different invasion pathways ${ }^{[57]}$.

As $P$. knowlesi has become an important Plasmodium species causing severe and fatal malaria in Southeast Asia, a comparative analysis of sequences and biochemical properties of three knowpains (KPs 2-4) and their VPs orthologs was conducted in India. Immunolocalization studies showed that KPs 2 and 3 , similar to VPs 2 and 3, were localized in the food vacuole. Knowpain-4 was present in the cytoplasm towards the parasite periphery, but was co-localized with hemozoin formation confirming its presence in the food vacuole as well. In addition, the investigators observed significant similarity between each KP and its VP ortholog, specially as regards their substrate specificity. Also, KP-4 was observed to have essential role in erythrocyte cytoskeleton breakdown facilitating merozoite egress. Accordingly, the investigators suggested using in vitro culture of $P$. knowlesi for searching a VP specific inhibitor in the absence of an efficient in vitro culture system for $P$. vivax ${ }^{[58]}$.

In a study conducted in Portugal, the investigators were able to immunolocalize chabaupain-1, a FP-1 ortholog in $P$. chabaudi, in mosquito ookinete stage, suggesting its relation to sporozoites egress from mosquito midgut. BALB/c mice immunized with the recombinant chabaupain-1 alone or in conjugation with chitosan nanoparticles as an adjuvant, showed partial protection when challenged with P. chabaudi ${ }^{[59]}$.

7. Expression system: Cuban investigators reviewed techniques utilized for expression systems in the previous studies to yield sufficient productive amounts of recombinant plasmodial CPs. They found that the majority displayed low productivity 
with high formation of insoluble aggregates. Their analysis revealed significant divergences in codon and nucleotide composition from those of $E$. coli bacterial genes. In addition, they demonstrated that genes encoding plasmodial CPs were distant from highly expressed genes in E. coli. They attributed this obstacle to the significant divergence in codon usage and $\mathrm{A}+\mathrm{T}$ content between the target gene (CPs) and the selected host (E. coli). This divergence usually leads to several obstacles in cloning techniques such as decreased mRNA stability, early termination of transcription and translation; occurrence of insertions and deletions and finally inhibition of protein synthesis or even arrest of cell growth. To obtain soluble and active products representing the structural and functional characteristics of the natural CPs, the investigators recommended replacing codons, rarely found in highly expressed $E$. coli genes, with more favorable codons found in the whole amino acids sequence of the target cloned CP. Accordingly, they suggested non-bacterial expression system instead of $E$. coli, e.g. yeasts such as Pichia pastoris and Saccharomyces cerevisiae $(S$. cerevisiae $]^{[60]}$.

\section{B] Dipeptidyl aminopeptidases (DPAPs)}

They are papain CPs (clan CA, family C1) required to release dipeptides from the $\mathrm{N}$-terminal of protein substrates. $P$. falciparum genome showed three cathepsin $C$ like homologs that are expressed during the intra-erythrocytic cycle. MEROPS website identified the first two DPAPs (1 and 2) as single CP (dipeptidyl peptidase), while DPAP-3 was identified as dipeptidyl aminopeptidase ${ }^{[61]}$. DPAP-1 was identified and its enzymatic activity was characterized in 2004. Its proenzyme form was found to be accumulated in PV, and it was immunolocalized in the food vacuole suggesting its role in hemoglobin degradation. Truncation of the chromosomal DPAP-1-coding sequence showed that cytosolic DPAP-1 activity is essential for asexual proliferation. Figure $(2)^{[62]}$ presents steps of the process of hemoglobin degradation as follows: 1) the first initial cleavage that occurs at the hinge region of the $\alpha$-globin chain is mediated by the aspartyl proteinases (plasmepsins I, II, and IV); 2) globin chain is cleaved by multi-enzymatic activity of FPs 2,2 and 3 , plasmepsins I, II, and IV and a histo-aspartic protease; 3) globin short polypeptides (10-20 amino acids) are converted to small oligopeptides (5-10 amino acids) by a metalloprotease (falcilysin); 4) DPAP-1 cleaves oligopeptides into dipeptides. In this study, the investigators demonstrated that DPAP-1 released dipeptides from the $\mathrm{N}$ terminal of oligopeptides. They

$$
\begin{aligned}
& \text { Hemoglobin PLMs I, II, IV } \alpha \text {-Globin } \begin{array}{c}
\text { PLMs I, II, IV } \\
\text { FPs 2, 2, } \mathbf{3} \text { \& HÄP }
\end{array} \\
& \begin{array}{c}
\text { Short } \\
\text { oligopeptides _FLC_oligopeptides }
\end{array} \\
& \begin{array}{l}
\text { (10-20 AA) } \\
\text { (5-10 AA) }
\end{array}
\end{aligned}
$$

Fig. 2. Proteinases contributing in hemoglobin degradation AA: amino acids, DPAP1: dipeptidyl aminopeptidase 1 , FLC: falcilysin, FPs: falcipains, HAP: histo-aspartic protease, PLMs: plasmepsins. Cited from Klemba et al. ${ }^{[62]}$. attributed failure to detect DPAP-1 activity in vitro in food vacuole to the lack of exogenously added reducing agent that is essential to prevent in vitro oxidative inactivation of DPAP-1 ${ }^{[62]}$. Similar to other papain family $\mathrm{CPs}$, the C-terminal of DPAPs have an extension motif to bind with its substrate; 8 for DPAP-1 and 20 amino acids for DPAPs 2 and $3^{[39]}$. Similar to other plasmodial CPs, this extension motif was suggested as a novel antimalarial drug target ${ }^{[40]}$.

On the other hand, Plasmodium DPAP-3 enzymatic activity was characterized and in contrast to the previously studied DPAPs, removal of its internal prodomain was not required for its activation. Utilizing three microscopies; super resolution, time-lapse fluorescence, and immunoelectron; the investigators immunolocalized DPAP-3 to the apical organelles, closely associated with the neck of the rhoptries. Expression occurs early during schizogony just immediately before merozoites egress, and the investigators demonstrated negative implication in egress cascade as previously suggested. Instead, DPAP-3 was notably essential for efficient de novo RBCs invasion since DPAP-3 mutant merozoites failed to efficiently invade new RBCs. For confirmation, the investigators utilized flow cytometry assay to differentiate between intracellular merozoites from those extracellularly attached to RBC surface. Results revealed that DPAP-3 was involved in the initial attachment of egressed merozoites to RBCs surface. In this concept, RBC invasion presents a multistep process: 1) initial recognition of $\mathrm{RBC}$ receptors by adhesin proteins on merozoite surface; 2) tight attachment to RBC membrane; 3) directing merozoite apical end to RBC membrane; 4) active invasion mediated by actinmyosin with membrane invagination to form PV; and finally 5) closure of both membranes of RBC and PV. Accordingly, the investigators concluded involvement of DPAP-3 in the second step of RBCs invasion ${ }^{[63]}$.

\section{C] Calpain (CALP)}

It belongs to clan $\mathrm{CA}$, family $\mathrm{C} 2$, also known as 'specifictissues CPs' due to their cytoplasmic expression. They are implicated in several biological functions such as muscle activity, cell signaling, migration and attachment; as well as cell-cycle development, regulation and differentiation. They are either typical or atypical. The typical form lacks domain IV which mediates $\mathrm{Ca}^{2+}$ binding and consequently its activity, regulation and dimerization. $P$. falciparum genome revealed only one gene encoding atypical CALP, i.e. $\mathrm{Ca}^{2+}$ activated $\mathrm{CP}^{[61]}$. The role of apicomplexan CALPs in parasite egress was investigated with controversial results. They were first thought to be implicated in escape of merozoites from the PV by their proteolysisdependent mechanism ${ }^{[64]}$. In another report published in the year 2009, the investigators concluded that Plasmodium CALP utilized host cell CALP to facilitate escape from PV, but they failed to determine the precise mechanism ${ }^{[65]}$. Later, a third study suggested that it is the SERAs 5 and 6, and not CALP, that utilized 
host CALP-1 to unfasten the RBC cytoskeleton, leading to membrane collapse and parasite egress ${ }^{[66]}$.

In the same year (2009), American investigators characterized a calcium-activated CALP that is highly conserved in Apicomplexan and Alveolates. Failure to generate a clone of $P$. falciparum lacking CALP expression, using three different techniques, strongly suggested that it is essential for optimal viability of intra-erythrocytic blood stages. The investigators developed a knockdown system to identify the functions of $\mathrm{Ca}^{2+}$-activated CALP. The results revealed $40-60 \%$ growth defect with delayed ring trophozoite transformation, as well as inability to progress to $\mathrm{S}$ phase in their growth. The investigators suggested its use as drug target due to its marked divergence from mammalian CALPs ${ }^{[67]}$. One year later, Korean investigators identified PfCALP as a $46 \mathrm{kDa}$ protein. Using confocal microscopy, it was localized in the cytoplasm of all intra-erythrocytic stages. However, its particularly increased expression observed in late ring stages and early trophozoites, confirmed its role in cellular development, transformation, and differentiation ${ }^{[68]}$. Previously, PfCALP was thought to consist of three catalytic domains: two central (IIa and IIb) and one at the C-terminal (III). In the year 2013, Korean investigators showed that its active form had one central domain (IIa) that possessed residues of cysteine $\mathrm{e}^{1035}$, histidine $\mathrm{e}^{1179}$, and asparagine $^{1195}$ positioned close to one another; forming the appropriate orientation for its catalytic activity. The investigators suggested that identification of its catalytic monomeric subdomain (active site) can facilitate development for a specific inhibitor utilizing HTS. To confirm their results, the investigators conducted mutation studies for those amino acid residues ${ }^{[69]}$.

In cerebral malaria, Plasmodium CALP was found to be involved in the associated axonal injury. Expression of $P$. falciparum CALP and endogenous human inhibitor of calpain, calpastatin, in axonal injury was evaluated in two studies. In the first study, increased CALP activation was observed in the cytosolic extract of mice cerebral cortex, with decreased calpastatin levels. The investigators concluded that plasmodial CALPs mediated axonal injury in complicated cerebral malaria ${ }^{[70]}$. In the second study, the investigators analyzed their expression in post-mortem brain tissues obtained from patients who died due to cerebral malaria. The results were compared with malaria infected cases without neurological manifestations and with apparently healthy control. Results revealed significant nuclear CALP expression in neurons of cerebral malaria post-mortem cases. In contrast, host calpastatin expression was rarely observed in injured axons $^{[71]}$. On the other hand, a Korean study showed that heat shock protein-60 (HSP-60) was co-localized with PfCALP in both trophozoites and schizonts. The investigators succeeded in demonstrating the role of $P$. falciparum HSP- 60 on the enzymatic activity of plasmodial CALP. Using mizoribine as an efficient HSP-60 inhibitor, in higher concentrations it inhibited
PfCALP enzymatic activity and malarial growth compared to ALLN, a typical CALP inhibitor. These results suggested the significant interaction of HSP-60 with $P f$-CALP enzymatic activity ${ }^{[72]}$.

\section{D] Serine repeat antigens (SERAs)}

Before the $20^{\text {th }}$ century, the only Plasmodium SERAs known were SERAs 5 and 6 and were termed serine repeat antigen (SERA) and serine stretch protein (SERPH). They are synthesized as 113-126 kDa precursor proteins and expressed in PV of all intraerythrocytic stages. They possess a papain protease-like central domain flanked by two cysteine-rich domains. SERAs are unique proteinases, in which cysteine motif in some SERAs is replaced by an active-site serine residue. Experimental cysteine $\leftrightarrow$ serine substitution at the active catalytic site significantly reduced their proteolytic activity ${ }^{[73,74]}$.

There are nine genes encoding $P$. falciparum SERAs; six with serine residue (1-5 and 9) and three with cysteine residue (6-8). The first eight genes encoding SERAs 1-8 are tandemly arrayed on chromosome 2, while the last gene is on chromosome 9. To characterize expression and functions of the genes encoding SERAs, Australian investigators observed that genes located at the periphery of the cluster on chromosome 2 ; i.e. encoding SERAs 2, 3, 7 and 8 could be knocked out. In contrast, they failed to knockout genes located in the central region encoding SERAs 4-6, suggesting their essential role in the replication of intra-erythrocytic stages of the tested laboratory lines. Although gene encoding SERA-1 is located at the periphery, the investigators failed to disrupt it. However, they observed little increase in SERA-1 expression in mature stages of wild type parasites ${ }^{[75]}$.

It was observed that presence of these two types of SERAs form distinct phylogenetic groups not only within $P$. falciparum, but also across other species, suggests different biological roles for members of each type. To identify SERAs orthologs in other Plasmodium species, an evolutionary history of SERA gene family members was established. A phylogenetic tree was constructed from nucleotide and amino acid sequence data for 33 putative SERA homologs in seven different species; $P$. falciparum $P$. vivax, $P$. yoelii, $P$. chabaudi, $P$. knowlesi, $P$. vinckei, and $P$. reichenowi. Results enabled the investigators to 1 ) predict the missing genes in some species; 2 ) clarify the relationship between different $P$. falciparum SERAs especially SERAs 5 and 6; and 3) gain more data for both SERAs in Plasmodium spp. affecting rodents. The investigators suggested that these data would help researchers working in development of a vaccine candidate or novel anti-malarial drugs utilizing their essential roles in P. falciparum. The investigators concluded that SERAs family gene tree is a rooted tree with two distinct evolutionary groupings according to SERA residue; cysteine or serine. At least two SERAs of each type are present in each Plasmodium species, and those affecting rodents; $P$. yoelii, $P$. vinckei, and $P$. chabaudi have only four SERAs. They recommended 
further studies to answer several questions: 1) why do different species exhibit different numbers of SERA genes? 2) which SERA is responsible for a certain biological role across human and rodent species?; 3 ) what are the functional orthologs for SERAs 5 and 6 in

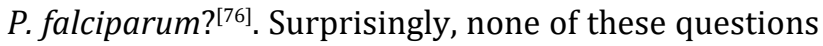
were answered in PubMed search till now.

As previously mentioned, PfSUB1 is localized in PV where it cleaves several PV proteins, among them is SERA- $6^{[49]}$. British investigators demonstrated that out of the three SERAs found in P. falciparum genome, only SERA-6 is an essential CP in intra-erythrocytic stages. They also identified three cleavage sites in SERA6 cleaved by SUB1 and this process was essentially required to convert SERA- 6 to an active $\mathrm{CP}^{[77]}$. Similar to other papain family plasmodial CPs, the C-terminal of SERAs have an extension motif (10 amino acids) to bind with its substrate ${ }^{[39]}$. Also, this extension motif was suggested as a novel anti-malarial drug target ${ }^{[40]}$.

\section{E] Metacaspases (MCAs)}

It is well known that MCAs are considered important drug targets due to their absence in humans. As previously discussed ${ }^{[78]}$, MCAs are CPs related to metazoan caspases and are involved in programmed cell death. Apoptosis is an essential host pathway contributing both innate and acquired immune responses. Plasmodium spp. as intracellular pathogens, have to act against cell apoptosis to protect them from cell death, and hence to survive and replicate. Therefore, strategy of "protozoa commit suicide to survive" was suggested ${ }^{[79]}$.

In the early $20^{\text {th }}$ centurey, Plasmodium genome database revealed two genes encoding MCA-like proteins $^{[4]}$. Four years later, Meslin et al. ${ }^{[80]}$ claimed that $P$. falciparum genome revealed three MCA genes, but only PfMCA-1 possesses the required histidine and cysteine proteolytic activity. The investigators suggested that decreased PfMCA-1 activity contributes, in a part, to Plasmodium drug resistance ${ }^{[80]}$. Accordingly, it was observed that blocking mechanisms involved in apoptosis can increase drug sensitivity and by-pass the common phenomenon of anti-malarial drug resistance. Previous studies observed that MCAs in intracellular pathogens such as $S$. cerevisiae ${ }^{[81]}$ and L. major ${ }^{[82]}$ induced apoptosis through a specific proteolytic activity. To investigate similar mechanisms conserved in all intracellular protozoa, e.g. P. falciparum, Meslin et al. $^{[83]}$ focused their study on the substrate specificity cleaved by plasmodial MCAs. Using complemented MCA-deficient yeast cells, the investigators compared the catalytic domain function of PfMCA-1, and LmMCA with S. cerevisiae MCA under oxidative stress conditions. As an example of a stress condition, the investigators utilized exposure of $P$. falciparum to anti-malarial drugs and use of z-VAD-fmk, as pan-caspase inhibitor. Results showed that the functional proteolytic activity of PfMCA-1 was a calcium-dependent activity, and arginine was the substrate specificity. In drug-treated parasites, results showed $82 \%$ growth inhibition compared to
$67 \%$ and $30 \%$ in L. major and S. cerevisiae, respectively, while using z-VAD-fmk produced apparent decreased apoptotic markers. The investigators concluded that PfMCA-1 showed potential role(s) to balance between life and death, and accordingly they suggested use of adjuvant or combined therapy of MCAs specific inhibitor with anti-malarial drugs ${ }^{[83]}$. The mentioned study denied earlier results of a study conducted to investigate contribution of PfMCA-1 in parasite growth and development. British investigators generated $P$. berghei parasites lacking gene encoding MAC-1. Results showed its expression only in female gametocytes and subsequent mosquito stages, but not in other intra-erythrocytic stages ${ }^{[84]}$. Recently in an Indian study, a second MCA was characterized in $P$. falciparum (PfMCA-2). The investigators demonstrated that its expression was evident in late schizonts and gametocytes, and general caspase inhibitors showed no effect on PfMCA-2 proteolytic activity ${ }^{[85]}$.

In a study conducted in Brazil, the investigators suggested utilizing the autophagic pathway as an alternative target for development of a novel antimalarial drug. Using light microscopy, the investigators assessed growth inhibition after in vitro pressure treatment with two anti-malarial drugs; chloroquine and staurosporine. When DNA fragmentations were observed, they added a culture pretreated with a general caspase inhibitor. Results revealed absence of apoptotic markers with observed cytoplasmic vacuolization detected by electron microscopy. Accordingly, the investigators suggested that an autophagy pathway, not apoptosis, was adapted by parasites under chloroquine or staurosporine pressure treatment ${ }^{[86]}$. Later, two other studies indicated that drug treatment induced Plasmodium MCAs activation. Using cell-based assays, the investigators showed increased mitochondrial permeability and significant increase of fragmented DNA associated with increased MAC activity after treatment with chloroquine and staurosporine ${ }^{[87]}$. Similar results were obtained when the investigators studied apoptotic markers and MCA activation in mefloquine-treated and untreated parasites. Significant production of reactive oxygen species $24 \mathrm{~h}$ after treatment was observed. The investigators concluded that mefloquine induced apoptosis through MCA activation and production of reactive oxygen species, beside its main mechanism of action, i.e. inhibition of hemozoin formation ${ }^{[88]}$.

\section{F] Ovarian tumor unit-like CP (OTU)}

A novel ovarian tumor unit domain-containing $\mathrm{CP}$ of $P$. falciparum (PfOTU) was recently identified. Using fluorescent affinity assay, Indian investigators showed its vesicular localization and its association with the apicoplast. Gene knockout showed abnormal apicoplast development which resulted in subsequent arrest of early-schizont developmental stages in further life cycles. It was observed that PfOTU is an essential CP in apicoplast protein import, and is significantly linked with apicoplast homeostasis ${ }^{[89]}$. 


\section{[II] Cysteine proteinase inhibitors (CPIs)}

According to their mechanism of action, reported FPs inhibitors are classified into four main categories: peptide, non-peptide, peptidomimetic, and recently allosteric site inhibitors. Besides, several herbal compounds (most commonly, non-peptide inhibitors) extracted from plant and fruits were investigated for their potent inhibitory activity against FPs. It is worth mentioning that although the majority of peptide inhibitors are the best in vitro FP-2 inhibitors, only one, the covalent inhibitor E-64, was obtained with a FP-2 crystal structure in a drug-like compound. However, peptide inhibitors were reported to give in vivo unsatisfactory results due to their poor pharmacological profiles and their susceptibility to degradation by host enzymes. Therefore, it was recommended to conduct structure-based screening studies to identify new non-peptide inhibitors competent with FP-2 crystal structure.

Two other approaches were utilized to search for a novel CPI, involving trials investigating known pure or synthesized compounds or their derivatives, and the screening of libraries for molecules with selective binding affinity to the target CP (virtual screening, VS). After discovery of FPs crystal structure, several studies were conducted utilizing structure-based recent technology. Pure or synthesized compounds were modified by addition, removal or replacement, to achieve better binding affinity and selectivity. Structurebased strategy is also applied in screening either using VS or HTS. Both are computational techniques used in drug discovery to search libraries, but the latter is more scientific as it utilizes data processing/control software, liquid handling devices, and sensitive detectors. Using HTS, researchers are able to quickly identify active compounds affecting certain biomolecular pathways ${ }^{[90]}$.

\section{A] Peptide inhibitors}

\section{Protease inhibitors}

a) Non-specific protease inhibitors: To characterize FPs structural activity relationship (SAR), an American study investigated phenyl vinyl sulfones, vinyl sulfonate esters, and vinyl sulfonamides as CPIs. Several compounds exhibited inhibitory activity on FPs and parasite growth. However, compounds containing leucine at the P2 position were more potent than those with phenylalanine at this position. There was a discrepancy in FPs SAR, i.e. no correlation between inhibitory activity of the target $\mathrm{FP}$ and growth inhibition. It was observed that some compounds exhibiting high activity against FPs showed poor inhibitory activity against parasite growth. However, vinyl sulfonate esters, and vinyl sulfonamides offered better inhibitory potency over phenyl vinyl sulfones ${ }^{[91]}$. Because FPs prefer leucine at P2 position in their substrates, a widely used proteasome peptidyl inhibitor, MG132, was selected. Results showed that MG132 in vitro inhibited parasite growth associated with food vacuole enlargement due to accumulation of undegraded hemoglobin, i.e. inhibition of hemoglobin degradation.
The investigators extended their studies to investigate its mechanism of action. Is the mechanism due to FPs inhibition or blockage of ubiquitin proteasomal system (UPS)? The later has an essential role in cellular protein turnover, and is responsible for tagging and removing damaged proteins, i.e. a degrading machine. Results revealed that MG132 blocked intra-erythrocytic survival and growth via inhibition of both $P$. falciparum enzymes (FPs and UPS). In addition, results showed over 200 times more potency and selectivity for malaria parasites than for human peripheral blood mononuclear cells. The investigators recommended further studies to design compounds possessing inhibitory potency against multi-enzyme targets of $P$. falciparum $^{[92]}$.

b) Specific DPAP inhibitors: In 2001, a study observed synergistic effects using a DPAP specific inhibitor; bestatin, combined with aspartyl inhibitors and/or other CPIs as elucidated by parasite growth ${ }^{[93]}$. Later, an American study investigated Ala-4(I)Phe-DMK for its potential inhibition of DPAP-1. It showed in vitro parasite death with 100-fold selectivity to DPAP-1 over other papain $P$. falciparum CPs (DPAP-3 and FPs) as well as 10 -fold sensitivity on trophozoites. The investigators demonstrated that sustained DPAP-1 inhibition for 2-3 $\mathrm{h}$ was required to prevent recovery of DPAP-1 activity. Performing in vivo studies confirmed that DPAP-1 inhibition resulted in clearance of parasitemia in two days after treatment. However, it showed toxicity to the mice at $20 \mathrm{mg} / \mathrm{Kg}$. The investigators validated DPAP as drug target and recommended further studies to design and synthesize new non-toxic DPAP inhibitor ${ }^{[94]}$.

c) Specific CALP inhibitors: The relatively selective inhibitor of human CALP-1 (BDA-410) was investigated as FPs inhibitor against recombinant FP-2 and native FPs in P. falciparum trophozoite extract. The American investigators also investigated its inhibitory effects on $P$. falciparum growth in vitro and on P. chabaudiinfected mice. BDA- 410 showed IC $_{50}$ values of 628 and 534 for recombinant FP-2`and native FPs, respectively. It also showed irreversible damage of in vitro intraerythrocytic stages and significant delay of in vivo malarial infection ${ }^{[95]}$.

Specific CALP inhibitor, ALLN, was investigated as anti-malarial drug. The in vitro study conducted in Korea showed that ALLN treatment in low concentration for $48 \mathrm{~h}$ inhibited CALP expression in all intra-erythrocytic stages, with significant eradication of parasitemia. The investigators recommended further in vivo studies ${ }^{[96]}$. Because ALLN has an aldehyde group that is susceptible for metabolism, another two Korean studies synthesized ALLN analogs with $\alpha, \beta$ unsaturated carbonyl peptides to act as electrophiles for CALP cysteine residues. Results revealed that three analogs were the most potent against intra-erythrocytic stages ${ }^{[97]}$. Also, synthesized compounds with two amino acids gave better results than those with one amino $\operatorname{acid}^{[98]}$ 
Three organotellurane derivatives were identified as potent selective CALP inhibitors. The derivatives (RF05, RF07 and RF19) exhibited potent in vitro antimalarial drugs activities against two $P$. falciparum sensitive and resistant strains. Because CALP is stimulated by calcium, the investigators confirmed CALP selectivity of the evaluated derivatives using an assay in the presence of calcium-ATPase inhibitor ${ }^{[99]}$.

d) Cysteine and serine protease inhibitors: Efficacy of five cysteine/serine inhibitors; PMSF, leupeptin, TPCK, TLCK, and 1,10-phenanthroline were in vitro evaluated on $P$. berghei gametogenesis and early zygote development. The first two compounds were inefficient. In contrast, the last three showed exflagellation inhibition at uM concentrations, while the last compound was the most effective. The results also showed a further reduction of ookinete production on addition of TPCK/TLCK at $2 \mathrm{~h}$ after gametogenesis. However, this reduction was not observed on addition of the last compound ${ }^{[100]}$. The effects of E-64, and that of cysteine/serine inhibitors (leupeptin and calpeptin) were investigated concerning egress cascade. All tested inhibitors blocked the final step of RBC membrane rupture in parasite egress. Calpeptin showed the most potent inhibitory effect. To demonstrate if the inhibitory effect is reversible or not, the investigators withdrew the tested inhibitors. Results showed that trapped schizonts restarted to release merozoites after RBC' membrane rupture ${ }^{[101]}$. New leupeptin analogs were designed and virtually screened utilizing structurebased designing approach to select the best inhibitors that selectively bind at the active site of FPs 2 and 3. Out of the 104 leupeptin analogs, the investigators detected 18 compounds; only three showed good binding affinity to FP-2 and the rest for FP-3. All the identified new analogs showed better binding than leupeptin itself, and those for FP-3 showed higher selectivity than those for FP-2. The investigators recommended future in vivo studies to investigate usefulness of leupeptin either alone or in combination with known anti-malarial drugs to achieve synergistic effective treatment $t^{[102]}$.

e) Cysteine and aspartic protease inhibitors: A group of Indonesian investigators synthesized six diaza-phenanthrene analogs to be tested as potential protease inhibitors of cysteine (FPs) and aspartic (plasmepsins) proteases that contribute in hemoglobin degradation. Only one compound showed a high in vitro potency against chloroquine-resistant and sensitive $P$. falciparum strains. Meanwhile, it showed high selectivity on $P$. falciparum proteases with very high $\mathrm{IC}_{50}$ value on Vero cells ${ }^{[103]}$. In addition, in vivo investigation showed clearance of parasitemia 4 days after treatment of $P$. berghei-infected mice ${ }^{[104]}$. To identify the mechanism of action, a third study was conducted by comparing the in vitro and in vivo results with those obtained using chloroquine and E-64. The investigators utilized an isobologram that described the cooperative inhibition of the selected compound in relation to that of E-64 and chloroquine. Curve results showed concave (in comparison to E-64) and slightly convex (in comparison with chloroquine) inclinations indicting its high potentiality as protease inhibitor ${ }^{[05]}$.

\section{Pure and synthesized compounds}

a) Sole inhibitors for FPs: Derivatives of several natural or synthesized compounds were investigated as CPIs for FP-2 alone or with FP-3. An American study evaluated the inhibitory activity of synthetic peptidyl aldehyde and $\alpha$-ketoamide as potent CPIs against FPs 2 and 3 as well as vinckepain-2. The tested compounds were also investigated as anti-malarial drugs against several $P$. falciparum strains. The lead compound (No. 9050), when investigated in vivo against $P$. vinckeiinfected mice, showed only delayed growth, but not complete parasite eradication $^{[106]}$. In a South African study, the investigators observed that thiolactonechalcones were more potent as anti-malarial drugs than isatin-chalcones. However, the later showed FP-2 inhibitory activity which was lost in thiolactonechalcones ${ }^{[107]}$. Results of an Indian study revealed that a compound, termed Ga6, showed $\mathrm{IC}_{50}$ of 3.1 and 1.1 $\mathrm{ug} / \mathrm{ml}$ against chloroquine-sensitive and resistant $P$. falciparum field isolates, respectively ${ }^{[108]}$. In Portugal, investigators recognized that several indolinones were moderate inhibitors of PfFP-2, but not FP-3, with efficient in vitro growth inhibition against chloroquineresistant isolates ${ }^{[109]}$.

Synthesized azadipeptide nitriles were produced. Their potential inhibitory activity on recombinant FPs 2 and 3 , as well as in vitro anti-malarial effects against chloroquine sensitive and resistant $P$. falciparum infected erythrocytes were evaluated. Two points were observed. First, the inhibitory activity of the tested compounds on recombinant FPs exceeded those on the parasites' growth, indicating involvement of other essential enzymes in parasite survival and growth. Second, variable results were obtained between compound structure and their potency against FPs activity and parasite growth. Compound 2 that has a leucine side chain in the P2 position showed the best FPs inhibition, while the corresponding phenylalanine derived analogue (compound 1) exhibited 10-fold less activity. Extension of the benzyl residue or its replacement by pentyl increased FP-2 binding affinity (compounds 4 and 5 versus 3). Similar to compound 1 , carbon analogs compounds ( 6 and 7 ) exhibited low inhibitory potency. Considering the requirements for parasite growth, it appeared that: 1) compounds 4 and 5 displayed the best activity; 2) all tested compounds showed more or less similar IC $_{50}$ values against chloroquine sensitive and resistant strains; 3) there was no significant difference between compounds 1 and 2 ; 4) compounds 1-5 were more potent than carbon analogs ( 6 and 7). The investigators validated azadipeptide nitriles as a novel class of anti-malarial compounds. They also concluded that SAR data are significantly helpful in future designing of synthesized compounds as FPs inhibitors ${ }^{[110]}$. 
A series of 2,4 thiazolidinediones was synthesized and evaluated for FP-2 inhibition. Utilizing molecular docking studies, the investigators were able to determine their potential binding mode in FP-2 active sites. Moreover, most compounds showed anti-malarial activity with low micro-molar concentrations against a $P$. falciparum resistant strain $^{[111]}$. Phthalimides functionalized with cyclic amines were synthesized, and evaluated for their in vitro FP-2 inhibition. Six compounds showed inhibitory activity, and two of them (No. 14 and 24) were identified as potent anti-malarial agents against chloroquine resistant strains ${ }^{[112]}$.

In a recent study, investigators focused on structural characterization of FPs prodomain and its interaction with FPs catalytic domain. To achieve this, they compared the structure of FPs and their orthologs with human cathepsins. Results showed that the prodomain of all plasmodial CPs possess amino acids residues (a refolding domain, figure 1) to mediate its endogenous inhibitory effect on its catalytic domain. This domain is required for correct folding of the mature domain to an active protease. Utilizing a continuous prodomain epitope mimicking strategy, they designed a peptide inhibitor which binds more strongly to plasmodial proteases than the human cathepsins. The investigators used CABS-dock web server to accurately demonstrate the binding pockets of the designed new peptide inhibitor. Chemical optimization of the designed inhibitor provided derivatives with better potency and selectivity as well as enhancement of its bioavailability and stability. Although the investigators believed that peptide inhibitors are not practical solutions as antimalarial drugs, they recommended future studies focusing on FPs prodomain ${ }^{[113]}$.

b) Dual actions: To obtain potential anti-malarial drug with dual action, investigators synthesized compounds conjugating cinnamic acid with a heterocyclic core known to prevent hemozoin formation. Using molecular modeling calculations, the investigators observed that small compounds with their vinyl bonds close to the catalytic cysteine of FPs were potent peptide inhibitors ${ }^{[14]}$. Also, the majority of new synthesized artemisinin derivatives exhibited in vitro inhibitory potency against $\mathrm{FP}-2^{[115]}$.

\section{Screening technology}

a) Sole inhibitors for FPs: An American study conducted a structure-based VS of a library against FP-2 crystal structure utilizing the Glide docking program. The investigators succeeded to identify twenty-one hits with potent FP-2 inhibitory activity. Among them, few compounds showed no selectivity when screened against homologous human $\mathrm{CPS}^{[116]}$. South African investigators conducted VS of the ZINC database, a library of commercially available compounds. Nineteen compounds out of the most promising sixty hits showed inhibitory potency against in vitro parasite growth of chloroquine resistant $P$. falciparum strain. In addition, the most active compound against cultured parasites also exhibited inhibitory potency against FP-2 activity, but with 50-times less activity. The investigators suggested that its primary target was not FP-2 ${ }^{[117]}$.

To develop an efficient anti-malarial drug for clinical trials, Indian investigators achieved their objective by conducting four studies. Utilizing docking studies, the investigators demonstrated the key residues (asparagine ${ }^{281}$, cysteine ${ }^{283}$, valine ${ }^{396}$ and aspartate $\left.{ }^{398}\right)$, responsible for binding between E-64, as inhibitor, and VP-2. Screening the 3D structure of VP-2 using three public available databases enabled them to identify three new CPIs. Then, they modified the identified CPIs according to the detected key residues. Finally, they found that the developed CPIs showed satisfactory "ADMET properties (absorption, distribution, metabolism, excretion and toxicity)"[118]. Tripeptide molecules were identified using structurebased in silico VS. To analyze SAR data of the identified compound, the investigators synthesized several derivatives with various substitutions in four positions. Results revealed that compound 74 showed potent inhibitory activity against in vitro parasite growth that corresponded with its inhibitory activity against FP-2. To validate compound 74 as novel anti-malarial drug, the investigators recommended in vivo studies ${ }^{[119]}$.

The year 2017 introduced the first computational study to predict the binding modes of FP-2 inhibitors according to the obtained SAR data of previous studies conducted for peptide inhibitors. The investigators proposed a new strategy to identify the molecular demands of a compound to efficiently bind to FP-2 in one complex ${ }^{[120]}$. One year later, the same group of investigators identified a promising novel anti-malarial drug (HTS07940). First, they utilized HTS to identify potential FP-2 inhibitors. Second, they conducted in vitro growth assays for the top-scoring compounds. Third, they performed docking studies and free energy calculations to evaluate the interaction of the best hits with FP-2 and human cathepsin K. Finally, they evaluated their cytotoxicity against HeLa cells. Two compounds (HTS07940 and HTS08262) were identified as FP-2 inhibitors, but the first was better for two reasons; lower $\mathrm{IC}_{50}$ against multidrug-resistant P. falciparum strain (2.91 versus $34 \mu \mathrm{M})$, and lower cytotoxicity ( 133 versus $350 \mu \mathrm{M})^{[121]}$.

On the other hand, only one study was conducted for VP-3 inhibitors. Due to unavailability of VP-3 crystal structure, a homology model was created guided by VP-3 pharmacophore, i.e. description of the molecular features necessary for the recognition of its specific substrate. Indian investigators conducted a structurebased VS and identified fourteen compounds that mapped with VP-3 core pharmacophore features. Molecular dynamic simulation and docking studies filtered them to only five lead compounds, while in silico ADMET and in vitro studies clearly identified only three compounds. Based on the obtained results, the investigators recommended further in vivo studies to validate these compounds as potential novel antimalarial drugs ${ }^{[122]}$. 
b) Inhibitors for FPs and other enzymes: Screening a library of synthesized thio-semicarbazones identified promising several leading compounds that showed high activity against the major CPs in P. falciparum, T. brucei, and T. cruzi; FP-2, rhodesain and cruzain, respectively. Cytotoxicity studies in mice revealed that only one compound showed observable toxicity after $62 \mathrm{~h}^{[123]}$. On the other hand, screening inhibitory compounds with dual activity on PfFP-2 and PfDHFR (dihydrofolate reductase, an essential enzyme for $P$. falciparum growth) enabled Chinese investigators to design and synthesize six compounds. One of them showed moderate in vivo anti-malarial activity in a dose dependent manner, with better safety and survival rate in infected mice than that of tested comparable anti-malarial drug ${ }^{[124]}$. Five years later, another Chinese group of investigators identified a novel series of compounds possessing double inhibitory activity against the previously mentioned plasmodial enzymes. Compound 24 exhibited the best in vitro and in vivo potency with $\mathrm{IC}_{50}$ in nanomolar concentrations against $P$. falciparum chloroquine-sensitive strains and $P$. berghei-infected mice, respectively. In addition, it showed moderate inhibition against $P$. falciparum chloroquine-resistant strain ${ }^{[125]}$.

\section{B] Non-peptide inhibitors}

1. Sole inhibitors for FPs: An American study utilized in silico VS of the chemical directory library $(\sim 355,000$ compounds) to discover 22 CPIs. Among them, eight compounds exhibited activity against FPs 2 and 3, with only one compound showing in vitro inhibition of intraerythrocytic stages with an $\mathrm{IC}_{50}$ of $9.5 \mathrm{uM}^{[126]}$. In 2009, three publications were reported by a Chinese group of investigators. They observed that non-peptidic FP-2 inhibitors that bind non-covalently to FP-2 can retain their potentiality for high in vivo activity and selectivity. Utilizing two different docking methods and VS of 81 compounds enabled investigators to identify 28 non-peptidic molecules as potential FP-2 inhibitors. One of them (compound 1) showed in vitro activity against intra-erythrocytic stages, with $\mathrm{IC}_{50}$ of $2.4 \mathrm{uM}^{[127]}$. Further, the investigators modified the identified compound and conducted SAR studies to emphasize SAR impact in improving development of a potent active and selective FP- 2 inhibitor. They designed and synthesized new twelve analogs (2A-L) and investigated their potentiality as FP-2 inhibitors. Results revealed potency of three compounds (2B, $2 \mathrm{~K}$ and $2 \mathrm{~L})$, and one of them $(2 \mathrm{~K})$ showed potency $\sim 3$ times greater than the original compound. Obtained SAR data revealed that all analogs, with exception of $2 \mathrm{~K}$, possess one $\mathrm{H}$-bond binding with the phenyl ring in $\mathrm{C}$ region of FP-2. It was found that SAR data attributed the high potency of $2 \mathrm{~K}$ to its possession of two $\mathrm{H}$ bonds that enhanced its molecular bioactivity ${ }^{[128]}$. In their third publication, the investigators designed and investigated fourteen compounds (2A-E, 3A-C, and 4AF) as FP-2 inhibitors. Results revealed high inhibitory effects with double increased potency for analog $2 \mathrm{~A}$ than in the original compound. SAR results revealed three observations: 1) replacement of allyl group with cyclohexyl improved potency of compound 2A; 2) replacement of phenyl group with cyclohexyl lost potency of compounds 3A-C; 3) N-substituents of the amide group decreased its toxicity. This study also confirmed the importance of SAR data in designing and development of novel non-peptidic FP-2 inhibitors ${ }^{[129]}$.

2. Dual action: Recently, eighteen novel benzimidazole acrylo nitriles derivatives were designed, synthesized and investigated as non-peptide FP-2 inhibitors as well as inhibitors of hemozoin formation. Only three compounds (R-01, R-04, and R-08) showed in vitro high potency against $P$. falciparum chloroquine sensitive and resistant strains and efficiently inhibited hemozoin formation with low cytotoxicity for Vero cells. Therefore, the investigators concluded that development of a new anti-malarial drug with dual action (inhibition of FP-2 and hemozoin formation) can be utilized in chloroquine resistant areas, as its mechanism of action is inhibition of hemozoin formation ${ }^{[130]}$.

\section{C] Peptidomimetic inhibitors}

As previously reviewed ${ }^{[78]}$, compounds supplementing the host with nitrous oxide (NOdonors) inactivate CPs. Italian scientists investigated the effects of SNO-102 on CP activities of P. falciparum, T. cruzi and L. infantum and showed that NO-donors are efficient CPIs, in a dose- and time-dependent manner. Furthermore, the results revealed that FP-2 inactivation was prevented and reversed by addition of dithiothreitol and L-ascorbic acid. Therefore, the investigators attributed FP-2 inactivation to NOmediated S-nitrosylation of the cysteine ${ }^{25}$ catalytic residue $^{[131]}$. In a similar manner, 'Michael acceptors' inactivate CPs through the combination of 'Michael donors' to $\mathrm{CP}$, forming a double stabilized carbon nucleophile. Another two Italian studies used synthesized compounds utilizing 1,4-benzodiazepine scaffold on an electrophilic vinyl sulfone moiety. Results revealed that two synthesized compounds showed significant selective inhibition of FP-2 as well as in vitro efficiency against intra-erythrocytic stages ${ }^{[132,133]}$. A set of 'Michael acceptors' utilizing etacrynic acid as the lead structure were screened for their inhibitory potency on recombinant FPs 2 and 3. The lead compounds were investigated in vitro against chloroquine-sensitive and resistant $P$. falciparum strains. Compound 24 was identified as the best FPs inhibitor. The investigators recommended future studies to evaluate the affinity binding properties of the identified compound, and to synthesize further peptidomimetic CPIs with etacrynic acid amides ${ }^{[134]}$.

Pyrimidine tetraoxane showed potent nanomolar activity against FP-2 as well as in vitro P. falciparum strains. It decreased parasitemia and increased survival rate of $P$. berghei-infected mice ${ }^{[135]}$. Furthermore, out of 22 designed and synthesized compounds, only one (homophenylalanine-based $\alpha$-hydroxyketone) 
showed potent inhibition of both FPs 2 and 3 activities with in vitro parasite killing activity ${ }^{[136]}$. Because peptidomimetic nitriles efficiently inhibit CPs by formation of a covalent reversible bond, a study was conducted to design new series of nitriles utilizing a structure-based molecular modelling approach. With exception of compounds 7 and 8, efficient binding affinity to $P$. falciparum FP-2 was observed. Interestingly, all compounds were inactive against human serine protease ( $\alpha$-chymotrypsin) indicating their selectivity to inhibit only $\mathrm{CPs}^{[137]}$.

\section{D] Allosteric site inhibitors}

Allosteric inhibitors are compounds capable of binding at a site other than the enzyme active site to change the catalytic activity of the enzyme. These compounds are more selective and potent; moreover, gene mutation never occurs in presence of high drug doses because these inhibitors don't alter the active site. Investigated allosteric inhibitors are either of peptide or non-peptide category. The most common drug used in treatment of African sleeping sickness, suramin, showed a potent allosteric inhibitory efficiency on FP-2 catalytic activity ${ }^{[138]}$. Combined structural biology tools and mass spectrometry enabled investigators to characterize the allosteric mechanism for inhibition of new non-peptidic organic compounds against FP-2 activity. The investigators demonstrated that compound 66 is the best non-competitive inhibitor that showed allosteric binding between FP-2 catalytic site and the hemoglobin binding $\operatorname{arm}^{[139]}$. As previously reported, certain residues at the interface of FPs pro- and mature domain are essentially required for prodomain dissociation and FPs auto-processing (hot spot) ${ }^{[38]}$. To specifically block the hot spot, an Indian study synthesized six aza-peptide compounds to be investigated as allosteric site inhibitors. Two compounds (1 and 3) showed high binding affinity at the hot spot causing prodomain rigidity and subsequent failure of structural reorganization. Both compounds arrested in vitro parasite growth due to FPs inhibition ${ }^{[140]}$.

\section{E] Herbal inhibitors}

1. Sole inhibitors for FPs: Plant-derived biologically active compounds were also investigated for selective potent FP-2 inhibitory activity. Annonaceous extracts, found in tropical and subtropical trees, showed promising results as anti-cancer drug and pesticidal activities. Several extracts were investigated and results revealed modest FP-2 inhibition with high activity against $P$. falciparum in vitro ${ }^{[141]}$. Investigation of symplostatin 4 (Sym4), a cyanobacterial secondary metabolite, showed high potency as anti-malarial drug. Besides, due to its biological activity and structural feature, the investigators demonstrated evidence that Sym 4 can act as FPs inhibitor. To achieve this, they synthesized several Sym 4 analogs that were analyzed using competitive activity-based protein profiling against $P$. falciparum FPs. Results revealed highest inhibitory potency against FPs 2 and 2 ', followed by FP-3 and FP-1. Analyzing SAR data, it was observed that replacement of methyl-methoxypyrrolinone (mmp) group at its C-terminal end by alanine, resulted in significant loss of its inhibitory potency against both FP-2 and parasite growth ${ }^{[142]}$. Indian investigators observed that licochalcone, isolated from Chinese licorice, proved its promising efficiency against $P$. falciparum. Synthesized 88 chalcones were investigated for their anti-malarial activity. Only five compounds showed in vitro satisfactory results against chloroquine-sensitive and resistant $P$. falciparum strains, as well as in vivo increased survival time of $P$. yoelii-infected mice. In addition, molecular docking studies showed that only 3 compounds had moderate FP-2 inhibitory activity ${ }^{[143]}$.

Structure-based VS of in-house natural products database identified ten natural compounds with moderate inhibitory activity against FP-2. One of them exhibited in vitro anti-plasmodial activity against $P$. falciparum chloroquine-sensitive and resistant strains $^{[144]}$. In silico VS was conducted to screen a small South African library including 23 natural compounds. The investigators utilized 3D structure docking approach against FPs 2 and 3 of $P$. falciparum, $P$. vivax, $P$. knowlesi and FP-2 of P. berghei, P. chabaudi and P. yoelii, as well as against two human cathepsins (total $11 \mathrm{CPs}$ ). Only one potential hit, 5PGA obtained from Capnella thyrsoidea, was identified. The investigators extended their study to conduct another search on ZINC database to identify 186 5PGA-like compounds. Calculation of 'docking energy' enabled the investigators to identify only five 5PGA analogs that were further analyzed using molecular dynamics against the tested CPs. Only three analogs showed promising inhibitory potency as well as significant selectivity between plasmodial and human CPs. Based on these results, the investigators recommended further similar researches to identify non-peptide FPs inhibitors from natural herbs followed by in vitro and in vivo studies ${ }^{[145]}$.

Neolignans, found in Brazilian plants were selected based on previous reports as antibacterial, antifungal, as well as on their efficiency in treatment of several parasitic diseases including malaria. Nine derivatives were synthesized and investigated in vitro against a chloroquine resistant $P$. falciparum strain and human hepatoma cells. Intermolecular interaction with FP-2 using molecular docking was also conducted. Two derivatives (No. 9 and 10) showed significant in vitro activity with no cytotoxicity effects. For the molecular docking results, the investigators demonstrated that both derivatives effectively occupied cavities S1 and S2 in FP-2 ${ }^{[146]}$. Coumarin containing pyrazoline derivatives were also synthesized. Results showed that one of them exhibited potent inhibitory binding to FP-2 as well as inhibition of in vitro growth of $P$. falciparum chloroquine-sensitive and resistant strains and in vivo $P$. berghei infected mice ${ }^{[147]}$. 
2. Dual action: Myricetin and fisetin are plant pigments commonly derived from vegetables, fruits and nuts. They have similar structure and antioxidant properties. A Chinese study showed their inhibitory activity on both FP-2 and plasmepsin II ${ }^{[148]}$. VS of a number of herbal compounds was utilized to identify dual inhibitors of P. falciparum FP-2 and plasmepsin II. Out of 292 screened phytochemicals, Indian investigators selected 46 compounds according to ADMET properties. Among them, only eight compounds showed potential binding affinity to the target CPs active sites, while compound 117 extracted from Millettia usaramensis showed the maximum efficiency ${ }^{[149]}$. Recently, a cystatin from sugarcane (Cane CPI-4), exhibited in vitro inhibitory potency against $P$. falciparum infected erythrocytes and recombinant FPs 2 and 3, and it decreased hemozoin formation and parasitemia ${ }^{[150]}$.

\section{[III] Cystatins (CYSs)}

The first report of identification and characterization of the endogenous CYS of P. falciparum FPs was in the year 2006. The investigators localized falstatin in the periphery of rings and early schizonts. It was diffusely expressed in late schizonts and egressed merozoites, but was absent in trophozoites where maximal FPs activity is not required. When falstatin was expressed in E. coli, it showed potent activity against FPs 2 and 3 , with weaker activity against FP-1 and DPAP-1. Treatment of late schizonts incubated with native and recombinant FPs 2 and 3 with falstatin antibodies blocked de novo RBCs invasion. The investigators concluded that falstatin is required to facilitate RBCs invasion via controlling FPs activity and/or preventing host CPs activity. They also suggested that when falstatin regulates FPs activity during de novo RBCs invasion, it allows selective activity by other proteases; i.e. serine and aspartyl, for efficient RBCs invasion ${ }^{[151]}$. Similarly, falstatin homologs of $P$. berghei ${ }^{[152]}$ and $P$. yoelii ${ }^{[153]}$ were identified and characterized. Beside their erythrocytic functions, both studies observed their essential role in facilitating sporozoites invasion in exoerythrocytic stages. The investigators immunolocalized falstatin homolog in the PV and the cytoplasm of hepatic schizonts of $P$. berghei. It had similar structure as falstatin and its biochemical analysis revealed its inhibitory potency against human cathepsin L-like CP as well as parasite CPs. The investigators suggested its ability to protect host cells from apoptosis, essentially required for completion of parasite hepatic development $\mathrm{t}^{[152]}$.

In contrast, a study conducted in Germany demonstrated the role of $P$. berghei falstatin homolog in sporozoites maturation inside the mosquito vector. In addition, the investigators showed that disruption of the gene encoding this function did not affect intraerythrocytic stages. Also, they demonstrated that absence of falstatin homolog was correlated with enhanced cleavage of CSP essential for sporozoites gliding motility and infectivity. Accordingly, the investigators suggested falstatin as a possible vaccine candidate $^{[154]}$. One year later, a study was conducted to elucidate this controversy. The investigators analyzed all the previous studies and observed important points: 1) CYS of the avian P. gallinaceum was expressed and secreted by salivary gland sporozoites only, while PbCYS was constitutively expressed in all stages; 2) failure attempts to knockout the gene encoding CYS in $P$. yoelii and $P$. falciparum, suggested its essential role in intra-erythrocytic stages. Therefore, a stage-specific knockout of the gene encoding falstatin homolog of $P$. berghei was generated to analyze its function in all life cycle stages. The investigators claimed that $P b C Y S$ is essentially for sporozoites gliding motility and its capability to invade both mosquito salivary glands and hepatocytes. However, only mild attenuation was observed in intra-erythrocytic stages. The investigators concluded that sporozoites motility is crucially required for salivary gland invasion and exoerythrocytic stages. Sporozoites require more motility and time to reach their target in mosquitoes and exoerythrocytic location rather than intra-erythrocytic stages, and that is where effects of CYS down regulation were more apparently observed ${ }^{[155]}$.

To elucidate interaction between FPs and their endogenous CYS, two studies were conducted by the same investigators. The first only described the recombinant production of the $\mathrm{C}$ terminal part of berghepain-2 endogenous CYS in a complex with FP-2 and its homologs in seven Plasmodium spp. In the second study, the investigators identified the crystal 3D structure of the C-terminal inhibitory domain of $P$. berghei endogenous CYS. Results showed differences in the binding mode when compared with CPIs of other papain family members including host-cell cathepsin B-like proteases. Mutational studies suggested that C-terminal possessed a motif, consisting of three loops termed $\mathrm{BC}, \mathrm{DE}$, and FG that specifically participate in FPs inhibition ${ }^{[156,157]}$. Later, utilizing site-directed mutagenesis, hemoglobin hydrolysis assays and peptide inhibition studies, Indian investigators demonstrated that only the first loop (BC) specifically inhibits FPs and VPs via hydrogen bonds. This loop was suggested as a drug target for development of an effective potent antimalarial novel CPI ${ }^{[158]}$.

\section{CONCLUDING REMARKS}

1. P. falciparum genome revealed presence of four falcipains (FPs) as potent hemoglobinases, with exception of FP-1. Because the latter was found to be associated with oocyst production, it was suggested as a new strategy for control of malaria transmission. On the other hand, other proteases contribute with FPs 2, 2 ' and 3 in hemoglobin degradation including DPAP-1 (cysteine), plasmepsins I, II and $\mathrm{V}$ (aspartyl), and falcilysin (metallo). Knockout of the gene encoding FP-3 was lethal suggesting its essential role in Plasmodium life cycle stages.

2. All FPs have a unique structural-functional relationship: 1) a $\mathrm{C}$ terminal hemoglobin binding 
domain and a $\mathrm{N}$ terminal refolding domain; 2) its prodomain has four small subdomains; three (cytosolic, transmembrane, luminal) for food vacuolar and endoplasmic reticulum trafficking, and an inhibitory subdomain; 3) hot spot interactions at the interface of pro- and mature domains are essential for FPs auto-processing.

3. The $\mathrm{C}$ terminals of all plasmodial CPs have an extension motif to specifically bind with its selective substrate. Amino acids in these motifs were identified: 8, 10, 10, 14 and 20 amino acids for DPAP-1, SERAs, FP-3, FP-2 and DPAPs 2 and 3, respectively. They are highly suggested as novel anti-malarial drug targets.

4. Using its proteolytic activities and to facilitate parasite egress, FP-2 has an essential role in cleavage of RBCs membrane ankyrin, while FP-3 role is in gamete egress. Other proteases contribute with FPs 2 and 3 in egress cascade and de novo RBCs invasion including DPAP-3 and SERAs 5 and 6 (cysteine) and SUBs (serine). Egress cascade includes sporozoite egress from Anopheles midgut, as well as egress of exo- and intra-erythrocytic stages from hepatocytes and infected RBCs, respectively.

5. For FP-2, one homolog (VP) and several orthologs (vinckepains, berghepains, chabaupains and knowpains) were identified and characterized in $P$. vivax, $P$. vinckei, $P$. berghei, $P$. chabaudi, and $P$. knowlesi, respectively. All showed important differences from FP-2 as regard their substrate preference and inhibitor sensitivity and specificity.

6. For development of CPIs, several publications investigated peptide, non-peptide, peptidomimetic, and allosteric site inhibitors as well as herbal extracts. Researches on available known protease inhibitors or pure or synthesized compounds or their derivatives revealed variable results regarding FPs inhibitory potency, in vitro parasite growth and in vivo in infected mice. Similar results were also obtained with screening technology.

7. CPIs with dual action were also addressed. Beside CPI inhibitory potency against FP-2, compounds were synthesized or screened to inhibit plasmepsin II, or hemozoin formation, or dihydrofolate reductase enzyme (DHFR).

8. After discovery of FPs crystal structure, structurebased studies with SAR data enabled the investigators to gain more information regarding FPs/CPIs interaction: 1) FPs prefer leucine at P2 position in their substrates; 2) FPs have a methyl-methoxy-pyrrolinone (mmp) group at its C-terminal end that is essentially required to bind with its substrate; 3 ) compounds with two H-bonds enhanced its molecular bioactivity.

9. Endogenous $P$. falciparum CYS (falstatin) is essentially for sporozoites gliding motility and its capability to invade both mosquito salivary glands and hepatocytes. Its role in intra-erythrocytic stages was also identified, however, it was less apparently observed as with sporozoite motility.
10. Identification of falstatin crystal structure enabled the investigators to identify compounds targeting loop (BC) of the $C$ terminal specifically inhibits FPs.

Conflict of interest: There is no conflict of interest.

\section{REFERENCES}

1. Rosenthal PJ. Proteases of malaria parasites: new targets for chemotherapy: synopses. Emerg Infect Dis 1998; 4(1):49-57.

2. Rosenthal PJ. Cysteine proteases of malaria parasites. Int J Parasitol 2004; 34(13-14):14891499.

3. Rosenthal PJ. Falcipains and other cysteine proteases of malaria parasites. Adv Exp Med Biol 2011; 712:30-48.

4. Wu Y, Wang $\mathrm{X}$, Liu $\mathrm{X}$, Wang $\mathrm{Y}$. Data-mining approaches reveal hidden families of proteases in the genome of malaria parasite. Genome Res 2003; 13(4):601-616.

5. Pandey KC. Centenary celebrations article: cysteine proteases of human malaria parasites. J Parasit Dis 2011; 35(2):94-103.

6. Coppi A, Pinzon-Ortiz C, Hutter C, Sinnis P. The Plasmodium circumsporozoite protein is proteolytically processed during cell invasion. Exp Med 2005; 201(1):27-33.

7. Rosenthal PJ, Nelson RG. Isolation and characterization of a cysteine proteinase gene of Plasmodium falciparum. Mol Biochem Parasitol 1992; 51(1):143-152.

8. Eksi S, Czesny B, Greenbaum DC, Bogyo M, Williamson KC. Targeted disruption of Plasmodium falciparum cysteine protease, falcipain 1 , reduces oocyst production, not erythrocytic stage growth. Mol Microbiol 2004 Jul;53(1):243-250.

9. Sijwali PS, Kato K, Seydel KB, Gut J, Lehman J, Klemba $\mathrm{M}$, et al. Plasmodium falciparum cysteine protease falcipain-1 is not essential in erythrocytic stage malaria parasites. Proc Natl Acad Sci USA 2004; 101(23):8721-8726.

10. Le Roch KG, Zhou Y, Blair PL, Grainger M, Moch JK, Haynes JD, et al. Discovery of gene function by expression profiling of the malaria parasite life cycle. Science 2003;301: 1503-1508.

11. Silvestrini F, Lasonder E, Olivieri A, Camarda G, van Schaijk B, Sanchez M, et al. Protein export marks the early phase of gametocytogenesis of the human malaria parasite Plasmodium falciparum. Mol Cell Proteomics. 2010; 9:1437-1448.

12. Salas F, Fichmann J, Lee GK, Scott MD, Rosenthal PJ. Functional expression of falcipain, a Plasmodium falciparum cysteine proteinase, supports its role as a malarial hemoglobinase. Infect Immun 1995; 63(6):2120-2125.

13. Francis SE, Gluzman IY, Oksman A, Banerjee D, Goldberg DE. Characterization of native falcipain, an enzyme involved in Plasmodium falciparum 
hemoglobin degradation. Mol Biochem Parasitol 1996; 83(2):189-200.

14. Shenai BR, Sijwali PS, Singh A, Rosenthal PJ. Characterization of native and recombinant falcipain-2, a principal trophozoite cysteine protease and essential hemoglobinase of Plasmodium falciparum. J Biol Chem 2000; 275(37):29000-29010.

15. Pandey KC, Sijwali PS, Singh A, Na BK, Rosenthal PJ. Independent intramolecular mediators of folding, activity, and inhibition for the Plasmodium falciparum cysteine protease falcipain-2. J Biol Chem 2004; 279(5):3484-3491.

16. Pandey KC, Wang SX, Sijwali PS, Lau AL, McKerrow $\mathrm{JH}$, Rosenthal PJ. The Plasmodium falciparum cysteine protease falcipain-2 captures its substrate, hemoglobin, via a unique motif. Proc Natl Acad Sci USA 2005;102(26):9138-9143.

17. Pandey KC, Barkan DT, Sali A, Rosenthal PJ. Regulatory elements within the prodomain of Falcipain-2, a cysteine protease of the malaria parasite Plasmodium falciparum. PLoS One 2009; 4(5):e5694.

18. Wang SX, Pandey KC, Somoza JR, Sijwali PS, Kortemme T, Brinen LS, et al. Structural basis for unique mechanisms of folding and hemoglobin binding by a malarial protease. Proc Natl Acad Sci USA 2006; 103(31):11503-11508.

19. Korde R, Bhardwaj A, Singh R, Srivastava A, Chauhan VS, Bhatnagar RK, et al. A prodomain peptide of Plasmodium falciparum cysteine protease (falcipain-2) inhibits malaria parasite development. J Med Chem 2008; 51(11):31163123.

20. Subramanian S, Hardt M, Choe Y, Niles RK, Johansen $\mathrm{EB}$, Legac J, et al. Hemoglobin cleavage sitespecificity of the Plasmodium falciparum cysteine proteases falcipain-2 and falcipain-3. PLoS One 2009; 4(4):e5156.

21. Dasaradhi PV, Korde R, Thompson J, Tanwar C, Nag $\mathrm{T}$, Chauhan VS, et al. Food vacuole targeting and trafficking of falcipain-2, an important cysteine protease of human malaria parasite Plasmodium falciparum. Mol Biochem Parasitol 2007; 156(1):12-23.

22. Kerr ID, Lee JH, Pandey KC, Harrison A, Sajid M, Rosenthal PJ, et al. Structures of falcipain-2 and falcipain-3 bound to small molecule inhibitors: implications for substrate specificity. J Med Chem 2009; 52(3):852-857.

23. Sijwali PS, Rosenthal PJ. Gene disruption confirms a critical role for the cysteine protease falcipain-2 in hemoglobin hydrolysis by Plasmodium falciparum. Proc Natl Acad Sci USA 2004; 101(13):4384-4389.

24. Jeong JJ, Kumar A, Hanada T, Seo PS, Li X, Hanspal M, Chishti AH. Cloning and characterization of Plasmodium falciparum cysteine protease, falcipain-2B. Blood Cells Mol Dis 2006; 36(3):429435.
25. Singh N, Sijwali PS, Pandey KC, Rosenthal PJ. Plasmodium falciparum: biochemical characterization of the cysteine protease falcipain-2'. Exp Parasitol 2006; 112(3):187-192.

26. Subramanian S, Sijwali PS, Rosenthal PJ. Falcipain cysteine proteases require bipartite motifs for trafficking to the Plasmodium falciparum food vacuole. J Biol Chem 2007; 282(34):24961-24969.

27. Jani D, Nagarkatti R, Beatty W, Angel R, SlebodnickC, Andersen J, et al. HDP: a novel heme detoxification protein from the malaria parasite. PLoS Pathog 2008; 4(4):e1000053.

28. Chugh M, Sundararaman V, Kumar S, Reddy VS, Siddiqui WA, Stuart KD, et al. Protein complex directs hemoglobin-to-hemozoin formation in Plasmodium falciparum. Proc Natl Acad Sci USA 2013; 110(14):5392-5397.

29. Marques AF, Gomes PS, Oliveira PL, Rosenthal PJ, Pascutti PG, Lima LM. Allosteric regulation of the Plasmodium falciparum cysteine protease falcipain-2 by heme. Arch Biochem Biophys 2015; 573:92-99.

30. Klonis N, Crespo-Ortiz MP, Bottova I, Abu-Bakar $\mathrm{N}$, Kenny S, Rosenthal PJ, et al. Artemisinin activity against Plasmodium falciparum requires hemoglobin uptake and digestion. Proc Natl Acad Sci USA 2011; 108:11405-11410.

31. Ariey F, Witkowski B, Amaratunga C, Beghain J, Langlois AC, Khim $\mathrm{N}$, et al. A molecular marker of artemisinin-resistant Plasmodium falciparum malaria. Nature 2014;505:50-55.

32. Ashley EA, Dhorda M, Fairhurst RM, Amaratunga C, Lim P, Suon S, et al. Spread of artemisinin resistance in Plasmodium falciparum malaria. N Engl J Med 2014;371:411-423.

33. Ponsuwanna $\mathrm{P}$, Kochakarn T, Bunditvorapoom $\mathrm{D}$, Kümpornsin $\mathrm{K}$, Otto TD, Ridenour $\mathrm{C}$, et al. Comparative genome-wide analysis and evolutionary history of hemoglobin-processing and haem detoxification enzymes in malarial parasites. Malar J 2016; 15:51.

34. Xie SC, Dogovski C, Hanssen E, Chiu F, Yang $\mathrm{T}$, Crespo MP, et al. Hemoglobin degradation underpins the sensitivity of early ring stage Plasmodium falciparum to artemisinins. J Cell Sci 2016; 129(2):406-416.

35. Sijwali PS, Shenai BR, Gut J, Singh A, Rosenthal PJ. Expression and characterization of the Plasmodium falciparum hemoglobinase falcipain-3. Biochem J 2001; 360(2):481-489.

36. Kolla VK, Prasad R, Sayyad Z, Atul, Shah AY, Allanki $\mathrm{AD}$, et al. Independent amino acid residues in the S2 pocket of falcipain-3 determine its specificity for P2 residues in substrates. Mol Biochem Parasitol. 2015; 202(2):11-22.

37. Eksi S, Czesny B, van Gemert GJ, Sauerwein RW, Eling W, Williamson KC. Inhibition of Plasmodium falciparum oocyst production by membranepermeant cysteine protease inhibitor E64d. 
Antimicrob Agents Chemother 2007; 51(3):10641070.

38. Sundararaj S, Singh D, Saxena AK, Vashisht K, Sijwali PS, Dixit R, et al.. The ionic and hydrophobic interactions are required for the auto-activation of cysteine proteases of Plasmodium falciparum. PLoS One 2012;7(10):e47227.

39. Pandey KC, Dixit R. Structure-function of falcipains: malarial cysteine proteases. J Trop Med 2012; 2012: 345195.

40. Mishra M, Singh V, Singh S. Structural insights into key Plasmodium proteases as therapeutic drug targets. Front Microbiol. 2019; 10: 394.

41. Raphael P, Takakuwa Y, Manno S, Liu SC, Chishti AH, Hanspal M. A cysteine protease activity from Plasmodium falciparum cleaves human erythrocyte ankyrin. Mol Biochem Parasitol 2000; 110(2):259272.

42. Hanspal M, Dua M, Takakuwa Y, Chishti AH, Mizuno A. Plasmodium falciparum cysteine protease falcipain-2 cleaves erythrocyte membrane skeletal proteins at late stages of parasite development. Blood 2002; 100(3):1048-1054.

43. Dhawan S, Dua M, Chishti AH, Hanspal M. Ankyrin peptide blocks falcipain-2-mediated malaria parasite release from red blood cells. J Biol Chem 2003; 278(32):30180-30186.

44. Dasaradhi PV, Mohmmed A, Kumar A, Hossain MJ, Bhatnagar RK, Chauhan VS, et al. A role of falcipain-2, principal cysteine proteases of Plasmodium falciparum in merozoite egression. Biochem Biophys Res Commun 2005; 336(4):10621068.

45. Chandramohanadas R, Park Y, Lui L, Li A, Quinn $\mathrm{D}$, Liew $\mathrm{K}$, et al. Biophysics of malarial parasite exit from infected erythrocytes. PLoS One 2011; 6(6):e20869.

46. Aly AS, Matuschewski K. A malarial cysteine protease is necessary for Plasmodium sporozoite egress from oocysts. J Exp Med 2005; 202(2):225230.

47. Sologub L, Kuehn A, Kern S, Przyborski J, Schillig $\mathrm{R}$, Pradel G. Malaria proteases mediate insideout egress of gametocytes from red blood cells following parasite transmission to the mosquito. Cell Microbiol 2011; 13(6):897-912.

48. Hale VL, Watermeyer JM, Hackett F, Vizcay-Barrena G, van Ooij C, Thomas JA, et al. Parasitophorus vacuole poration precedes its rupture and rapid host erythrocyte cytoskeleton collapse in Plasmodium falciparum egress. Proc Natl Acad Sci USA 2017; 114(13):3439-3444.

49. Thomas JA, Tan MSY, Bisson C, Borg A, Umrekar TR, Hackett F, et al. A protease cascade regulates release of the human malaria parasite Plasmodium falciparum from host red blood cells. Nat Microbiol 2018; 3(4):447-455.

50. Desai PV, Avery MA. Structural characterization of vivapain-2 and vivapain-3, cysteine proteases from Plasmodium vivax: comparative protein modeling and docking studies. J Biomol Struct Dyn 2004;21(6):781-790.

51. Na BK, Shenai BR, Sijwali PS, Choe Y, Pandey KC, Singh A, et al. Identification and biochemical characterization of vivapains, cysteine proteases of the malaria parasite Plasmodium vivax. Biochem J 2004; 378(Pt 2): 529-538.

52. Na BK, Bae YA, Zo YG, Choe Y, Kim SH, Desai PV, et al. Biochemical properties of a novel cysteine protease of Plasmodium vivax, vivapain-4. PLoS Negl Trop Dis 2010; 4(10):e849.

53. Moon SU, Kang JM, Kim TS, Kong Y, Sohn WM, Na BK. Plasmodium vivax: collaborative roles for plasmepsin 4 and vivapains in hemoglobin hydrolysis. Exp Parasitol 2011; 128(2):127-132.

54. Singh A, Shenai BR, Choe Y, Gut J, Sijwali PS, Charles S. Craik CS, Rosenthal PJ. Critical role of amino acid 23 in mediating activity and specificity of vinckepain-2, a papain-family cysteine protease of rodent malaria parasites. Biochem J 2002; 368, 273-281.

55. Chan C, Goh LL, Sim TS. Differences in biochemical properties of the plasmodial falcipain-2 and berghepain-2 orthologues: implications for in vivo screens of inhibitors. FEMS Microbiol Lett 2005; 249(2):315-321.

56. Singh A, Walker KJ, Sijwali PS, Lau AL, Rosenthal PJ. A chimeric cysteine protease of Plasmodium berghei engineered to resemble the Plasmodium falciparum protease falcipain-2. Protein Eng Des Sel 2007; 20(4):171-177.

57. Hopp CS, Bennett BL, Mishra S, Lehmann C, Hanson $\mathrm{KK}$, Lin JW, et al. Deletion of the rodent malaria ortholog for falcipain-1 highlights differences between hepatic and blood stage merozoites. PLoS Pathog 2017; 13(9):e1006586.

58. Prasad R, Atul, Soni A, Puri SK, Sijwali PS. Expression, characterization, and cellular localization of knowpains, papain-like cysteine proteases of the Plasmodium knowlesi malaria parasite. PLoS One 2012: 7(12):e51619.

59. Armada A, Gazarini ML, Gonçalves LM, Antunes S, Custódio A, Rodrigues A, et al. Generation of an antibody that recognizes Plasmodium chabaudi cysteine protease (chabaupain-1) in both sexual and asexual parasite life cycle and evaluation of chabaupain-1 vaccine potential. Exp Parasitol 2013; 135(1):166-174.

60. Sarduy ES, Chávez-Planes ML. Efficient expression systems for cysteine proteases of malaria parasites: too good to be true? Bioengineered 2013; 4(2):107-114.

61. MEROPS. http://www.merops.sanger.ac.uk Release 11.0, last update: 6 June, 2019.

62. Klemba M, Gluzman I, Goldberg DE. A Plasmodium falciparum dipeptidyl aminopeptidase I participates in vacuolar hemoglobin degradation. J Biol Chem 2004; 279(41):43000-43007.

63. Lehmann C, Tan MSY, de Vries LE, Russo I, Sanchez MI, Goldberg DE, et al. Plasmodium falciparum 
dipeptidyl aminopeptidase 3 activity is important for efficient erythrocyte invasion by the malaria parasite. PLoS Pathog 2018; 14(5):e1007031.

64. Salmon BL, Oksman A, Goldberg DE. Malaria parasite exit from the host erythrocyte: a two-step process requiring extra-erythrocytic proteolysis. Proc Natl Acad Sci USA 2001; 98(1):271-276.

65. Chandramohanadas $\mathrm{R}$, Davis $\mathrm{PH}$, Beiting DP, Harbut MB, Darling C, Velmourougane G, et al. Apicomplexan parasites co-opt host calpains to facilitate their escape from infected cells. Science 2009; 324(5928):794-797.

66. Millholland MG, Chandramohanadas R, Pizzarro A, Wehr A, Shi H, Darling C, et al. The malaria parasite progressively dismantles the host erythrocyte cytoskeleton for efficient egress. Mol Cell Proteomics 2011; 10(12):M111.010678.

67. Russo I, Oksman A, Vaupel B, Goldberg DE. A calpain unique to alveolates is essential in Plasmodium falciparum and its knockdown reveals an involvement in pre-S-phase development. Proc Natl Acad Sci USA 2009; 106(5):1554-1559.

68. Choi YY, Jung SY, Cho PY, Soh BY, Zheng B, Kim SY, et al. Confocal microscopic findings of cysteine protease calpain in Plasmodium falciparum. Exp Parasitol 2010; 124(3):341-345.

69. Soh BY, Song HO, Lee Y, Lee J, Kaewintajuk K, Lee $\mathrm{B}$, et al. Identification of active Plasmodium falciparum calpain to establish screening system for Pf-calpain-based drug development. Malar J 2013; $12: 47$.

70. Shukla M, Rajgopal Y, Babu PP. Activation of calpains, calpastatin and spectrin cleavage in the brain during the pathology of fatal murine cerebral malaria. Neurochem Int 2006; 48(2):108-113.

71. Medana IM, Day NP, Hien TT, Mai NT, Bethell D, Phu NH, et al. Cerebral calpain in fatal falciparum malaria. Neuropathol Appl Neurobiol 2007; 33(2):179-192.

72. Yeo SJ, Liu DX, Park H. Potential interaction of Plasmodium falciparum HSP60 and calpain. Korean J Parasitol 2015; 53(6):665-673.

73. McCoubrie JE, Miller SK, Sargeant T, Good RT, Hodder AN, Speed TP, et al. Evidence for a common role for the serine-type Plasmodium falciparum serine repeat antigen proteases. Implications for vaccine and drug design. Infect Immun 2007; 75:5565-5574.

74. Arisue N, Kawai S, Hirai M, Palacpac NM, Jia M, Kaneko A, Tanabe K., Horii T. Clues to evolution of the SERA multigene family in 18 Plasmodium species. PLoS One 2011; 6, e17775.

75. Miller SK, Good RT, Drew DR, Delorenzi M, Sanders PR, Hodder AN, et al. A subset of Plasmodium falciparum SERA genes are expressed and appear to play an important role in the erythrocytic cycle. J Biol Chem 2002; 277(49):47524-47532.

76. Bourgon R, Delorenzi M, Sargeant T, Hodder AN, Crabb BS, Speed TP. The serine repeat antigen (SERA) gene family phylogeny in Plasmodium: the impact of GC content and reconciliation of gene and species trees. Mol Biol Evol 2004; 21(11):21612171.

77. Ruecker A, Shea M, Hackett F, Suarez C, Hirst EM, Milutinovic K, et al. Proteolytic activation of the essential parasitophorus vacuole cysteine protease SERA6 accompanies malaria parasite egress from its host erythrocyte. J Biol Chem 2012; 287(45):37949-37963.

78. Abaza SM. Expression of cysteine proteinases and cystatins in parasites and use of cysteine proteinase inhibitors in parasitic diseases. Part III: Protozoa (1). PUJ 2019; 12(1): 8-17.

79. Reece SE, Pollitt LC, Colegrave N, Gardner A. The meaning of death: evolution and ecology of apoptosis in protozoan parasites. PLoS Pathog 2011; 7(12): e1002320.

80. Meslin B, Barnadas C, Boni V, Latour C, De Monbrison F, Kaiser K, et al. Features of apoptosis in Plasmodium falciparum erythrocytic stage through a putative role of PfMCA1 metacaspaselike protein. J Infect Dis 2007; 195(12):1852-1859.

81. Madeo F, Engelhardt S, Herker E, Lehmann N, Maldener C, Proksch A et al. Apoptosis in yeast: a new model system with applications in cell biology and medicine. Curr Genet 2002; 41:208-216.

82. Zalila H, Gonzalez IJ, El-Fadili AK, Delgado MB, Desponds C, Schaff C et al. Processing of metacaspase into a cytoplasmic catalytic domain mediating cell death in Leishmania major. Mol Microbiol 2011;79:222-239.

83. Meslin B, Beavogui AH, Fasel N, Picot S. Plasmodium falciparum metacaspase PfMCA-1 triggers a z-VADfmk inhibitable protease to promote cell death. PLoS One 2011; 6(8):e23867.

84. Le Chat L, Sinden RE, Dessens JT. The role of metacaspase 1 in Plasmodium berghei development and apoptosis. Mol Biochem Parasitol 2007; 153(1):41-47.

85. Vandana, Singh AP, Singh J, Sharma R, Akhter M, Mishra PK, Saxena AK, et al. Biochemical characterization of unusual cysteine protease of $P$. falciparum, metacaspase-2 (MCA-2). Mol Biochem Parasitol 2018; 220:28-41.

86. Totino PR, Daniel-Ribeiro CT, Corte-Real S, de Fátima Ferreira-da-Cruz M. Plasmodium falciparum: erythrocytic stages die by autophagiclike cell death under drug pressure. Exp Parasitol 2008; 118(4):478-486.

87. Ch'ng JH, Kotturi SR, Chong AG, Lear MJ, Tan KS. A programmed cell death pathway in the malaria parasite Plasmodium falciparum has general features of mammalian apoptosis but is mediated by clan CA cysteine proteases. Cell Death Dis 2010; 1:e26.

88. Gunjan S, Singh SK, Sharma T, Dwivedi H, Chauhan BS, Imran Siddiqi $\mathrm{M}$, et al. Mefloquine induces ROS mediated programmed cell death in malaria parasite Plasmodium. Apoptosis. 2016; 21(9):955964. 
89. Datta G, Hossain ME, Asad M, Rathore S, Mohmmed A. Plasmodium falciparum OTU-like cysteine protease (PfOTU) is essential for apicoplast homeostasis and associates with non-canonical role of Atg8. Cell Microbiol 2017; 19(9).

90. Macarron R, Banks MN, Bojanic D, Burns DJ, Cirovic DA, Garyantes T, et al. Impact of high-throughput screening in biomedical research. Nat Rev Drug Discov 2011; 10(3): 188-195.

91. Shenai BR, Lee BJ, Alvarez-Hernandez A, Chong PY, Emal CD, Neitz RJ, et al. Structure-activity relationships for inhibition of cysteine protease activity and development of Plasmodium falciparum by peptidyl vinyl sulfones. Antimicrob Agents Chemother 2003; 47(1):154-160.

92. Prasad R, Atul, Kolla VK, Legac J, Singhal N, Navale $\mathrm{R}$, et al. Blocking Plasmodium falciparum development via dual inhibition of hemoglobin degradation and the ubiquitin proteasome system by MG132.PLoS One 2013; 8(9):e73530.

93. Gavigan CS, Machado SG, Dalton JP, Bell A. Analysis of antimalarial synergy between bestatin and endoprotease inhibitors using statistical responsesurface modelling. Antimicrob Agents Chemother 2001; 45(11):3175-3181.

94. Deu E, Leyva MJ, Albrow VE, Rice MJ, Ellman JA, Bogyo M. Functional studies of Plasmodium falciparum dipeptidyl aminopeptidase I using small molecule inhibitors and active site probes. Chem Biol 2010; 17(8):808-819.

95. Li X, Chen H, Jeong JJ, Chishti AH. BDA-410: a novel synthetic calpain inhibitor active against blood stage malaria. Mol Biochem Parasitol 2007; 155(1):26-32.

96. Jung SY, Zheng B, Choi YY, Soh BY, Kim SY, Park KI, Park H. Antimalarial effect of N-acetyl-LLeucyl-L-leucyl-L-norleucinal by the inhibition of Plasmodium falciparum calpain. Arch Pharm Res 2009; 32(6):899-906.

97. Mallik SK, Li DY, Cui M, Song HO, Park H, Kim HS. Synthesis and evaluation of peptidyl $\alpha, \beta-$ unsaturated carbonyl derivatives as anti-malarial calpain inhibitors. Arch Pharm Res 2012; 35(3):469-479.

98. Choi HJ, Cui M, Li DY, Song HO, Kim HS, Park H. Anti-malarial activity of new N-acetyl-L-leucyl-Lleucyl-L-norleucinal (ALLN) derivatives against Plasmodium falciparum. Bioorg Med Chem Lett 2013; 23(5):1293-1296.

99. El ChamyMaluf S, Melo PM, Varotti FP, Gazarini ML, Cunha RL, Carmona AK. Hypervalent organotellurium compounds as inhibitors of $P$. falciparum calcium-dependent cysteine proteases. Parasitol Int 2016; 65(1):20-22.

100. Torres JA, Rodriguez MH, Rodriguez MC, de la Cruz Hernandez-Hernandez F. Plasmodium berghei: effect of protease inhibitors during gametogenesis and early zygote development. Exp Parasitol 2005; 111(4):255-259.
101. Glushakova S, Mazar J, Hohmann-Marriott MF, Hama E, Zimmerberg J. Irreversible effect of cysteine protease inhibitors on the release of malaria parasites from infected erythrocytes. Cell Microbiol 2009; 11(1):95-105.

102. Kesharwani RK, Singh DV, Misra K. Computationbased virtual screening for designing novel antimalarial drugs by targeting falcipain-III: a structure-based drug designing approach. J Vector Borne Dis 2013; 50(2):93-102.

103. Sholikhah EN, Supargiyono S, Jumina J, Wijayanti MA, Tahir I, Hadanu $\mathrm{R}$, et al. In vitro anti-plasmodial activity and cytotoxicity of newly synthesized N-alkyl and N-benzyl-1,10phenanthrolinederivatives. Southeast Asian J Trop Med Public Health 2006; 37(6):1072-1077.

104. Wijayanti MA, Sholikhah EN, Hadanu R, Jumina J, Supargiyono S, Mustofa M. Additive in vitro antiplasmodial effect of $\mathrm{N}$-alkyl and N-benzyl-1,10phenanthroline derivatives and cysteine protease inhibitor E64. Malar Res Treat 2010; 2010:540786.

105. Wijayanti MA, Sholikhah EN, Tahir I, Hadanu R, Jumina J, Supargiyono S, et al. Anti-plasmodial activity and acute toxicity of N-alkyl and N-benzyl1,10-phenanthroline derivatives in mouse malaria model. J Health Sci 2006; 52(6):794-799.

106. Lee BJ, Singh A, Chiang P, Kemp SJ, Goldman EA, Weinhouse MI, et al. Antimalarial activities of novel synthetic cysteine protease inhibitors. Antimicrob Agents Chemother 2003; 47(12):3810-3814.

107. Hans RH, Gut J, Rosenthal PJ, Chibale K. Comparison of the anti-plasmodial and falcipain-2 inhibitory activity of beta-amino alcohol thiolactonechalcone and isatin-chalcone hybrids. Bioorg Med Chem Lett 2010; 20(7):2234-2237.

108. Wanare G, Aher R, Kawathekar N, Ranjan R, Kaushik NK, Sahal D. Synthesis of novel alphapyranochalcones and pyrazoline derivatives as Plasmodium falciparum growth inhibitors. Bioorg Med Chem Lett 2010; 20(15):4675-4678.

109. Praveen-Kumar SP, Gut J, Guedes RC, Rosenthal PJ, Santos MM, Moreira R. Design, synthesis and evaluation of 3-methylene-substituted indolinones as anti-malarials. Eur J Med Chem 2011; 46(3):927933.

110. Löser R, Gut J, Rosenthal PJ, Frizler M, Gütschow M, Andrews KT. Antimalarial activity of azadipeptide nitriles. Bioorg Med Chem Lett 2010; 20(1):252255.

111. Sharma RK, Younis Y, Mugumbate G, Njoroge M, Gut J, Rosenthal PJ, et al. Synthesis and structureactivity-relationship studies of thiazolidinediones as anti-plasmodial inhibitors of the Plasmodium falciparum cysteine protease falcipain-2.Eur J Med Chem 2015; 90:507-518.

112. Singh AK, Rajendran V, Pant A, Ghosh PC, Singh $\mathrm{N}$, Latha $\mathrm{N}$, et al. Design, synthesis and biological evaluation of functionalized phthalimides: a new class of anti-malarials and inhibitors of falcipain-2, 
a major hemoglobinase of malaria parasite. Bioorg Med Chem 2015; 23(8):1817-1827.

113. Musyoka TM, Njuguna JN, Bishop, ÖT. Comparing sequence and structure of falcipains and human homologs at prodomain and catalytic active site for malarial peptide based inhibitor design. Malar J 2019; 18: 159.

114.Pérez BC, Teixeira C, Figueiras M, Gut J, Rosenthal PJ, Gomes JR, Gomes P. Novel cinnamic acid/4-aminoquinoline conjugates bearing non-proteinogenic amino acids: towards the development of potential dual action antimalarials. Eur J Med Chem 2012; 54:887-899.

115. Liu Y, Lu WQ, Cui KQ, Luo W, Wang J, Guo C. Synthesis and biological activities of novel artemisinin derivatives as cysteine protease falcipain-2 inhibitors. Arch Pharm Res 2012; 35(9):15251531.

116. Shah F, Mukherjee P, Gut J, Legac J, Rosenthal PJ, Tekwani BL, et al. Identification of novel malarial cysteine protease inhibitors using structurebased virtual screening of a focused cysteine protease inhibitor library. J Chem Inf Model 2011; 51(4):852-864.

117. Mugumbate G, Newton AS, Rosenthal PJ, Gut J, Moreira R, Chibale K, Guedes RC. Novel antiplasmodial hits identified by virtual screening of the ZINC database. J Comput Aided Mol Des 2013; 27(10):859-971.

118. Yadav MK, Singh A, Swati D. A knowledge-based approach for identification of drugs against vivapain-2 protein of Plasmodium vivax through pharmacophore-based virtual screening with comparative modelling. Appl Biochem Biotechnol 2014; 173(8):2174-2188.

119. Chakka SK, Kalamuddin M, Sundararaman S, Wei $\mathrm{L}$, Mundra S, Mahesh $\mathrm{R}$, et al. Identification of novel class of falcipain-2 inhibitors as potential antimalarial agents. Bioorg Med Chem 2015; 23(9):2221-2240.

120.Hernández-González JE, Hernández Alvarez L, Pascutti PG, Valiente PA. Predicting binding modes of reversible peptide-based inhibitors of falcipain-2 consistent with structure-activity relationships. Proteins 2017; 85(9):1666-1683.

121. Hernández-González JE, Salas-Sarduy E, Hernández Ramírez LF, Pascual MJ, Álvarez DE, Pabón A, et al. Identification of (4-(9H-fluoren9-yl) piperazin-1-yl) methanone derivatives as falcipain 2 inhibitors active against Plasmodium falciparum cultures. Biochim Biophys Acta Gen Subj 2018; 1862(12):2911-2923.

122. Saddala MS, Adi PJ. Discovery of small molecules through pharmacophore modeling, docking and molecular dynamics simulation against Plasmodium vivax vivapain-3 (VP-3). Heliyon 2018; 4(5):e00612.

123. Greenbaum DC, Mackey Z, Hansell E, Doyle P, Gut J, Caffrey CR, et al. Synthesis and structure-activity relationships of parasiticidal thiosemicarbazone cysteine protease inhibitors against Plasmodium falciparum, Trypanosoma brucei, and Trypanosoma cruzi. J Med Chem 2004 3;47(12):3212-3219.

124. Huang H, Lu W, Li X, Cong X, Ma H, Liu X, et $a l$, Design and synthesis of small molecular dual inhibitor of falcipain-2 and dihydrofolate reductase as antimalarial agent. Bioorg Med Chem Lett 2012;22(2):958-962.

125. Chen W, Huang Z, Wang W, Mao F, Guan L, Tang $\mathrm{Y}$, et al. Discovery of new antimalarial agents: Second-generation dual inhibitors against FP-2 and PfDHFR via fragments assembly. Bioorg Med Chem 2017; 25(24):6467-6478.

126. Desai PV, Patny A, Gut J, Rosenthal PJ, Tekwani B, Srivastava A, et al. Identification of novel parasitic cysteine protease inhibitors by use of virtual screening. 2. The available chemical directory. J Med Chem 2006; 49(5):1576-1584.

127. Li H, Huang J, Chen L, Liu X, Chen T, Zhu J, et al. Identification of novel falcipain-2 inhibitors as potential antimalarial agents through structurebased virtual screening. J Med Chem 2009; 52(15):4936-4940.

128. Zhu J, Chen T, Chen L, Lu W, Che P, Huang J, et al. 2-amido-3-(1H-indol-3-yl)-N-substituted-propanamides as a new class of falcipain-2 inhibitors. 1. Design, synthesis, biological evaluation and binding model studies. Molecules 2009a; 14(1):494-508.

129.Zhu J, Chen T, Liu J, Ma R, Lu W, Huang J, et al. 2-(3,4-dihydro-4-oxothieno[2,3-d]pyrimidin-2ylthio) acetamides as a new class of falcipain-2 inhibitors. 3. Design, synthesis and biological evaluation. Molecules 2009b; 14(2):785-797.

130. Sharma K, Shrivastava A, Mehra RN, Deora GS, Alam MM, Zaman MS, et al. Synthesis of novel benzimidazole acrylonitriles for inhibition of Plasmodium falciparum growth by dual target inhibition. Arch Pharm (Weinheim) 2018; 351(1). DOI: $10.1002 / \operatorname{ardp} .201700251$.

131. Ascenzi P, Bocedi A, Gentile M, Visca P, Gradoni L. Inactivation of parasite cysteine proteinases by the NO-donor 4-(phenylsulfonyl)-3-((2-(dimethylamino)ethyl)thio)-furoxan oxalate. Biochim Biophys Acta 2004; 1703(1):69-77.

132. Ettari R, Nizi E, Di Francesco ME, Dude MA, Pradel G, Vicík R, et al. Development of peptidomimetics with a vinyl sulfone warhead as irreversible falcipain-2 inhibitors. J Med Chem 2008; 51(4):988-996.

133. Ettari R, Micale N, Schirmeister T, Gelhaus C, Leippe $\mathrm{M}$, Nizi E, et al. Novel peptidomimetics containing a vinyl ester moiety as highly potent and selective falcipain-2 inhibitors.102. J Med Chem 2009; 52(7):2157-2160.

134. Dude MA, Kaeppler U, Herb M, Schiller M, Schulz F, Vedder B, et al. Synthesis and evaluation of non-peptidic cysteine protease inhibitors of $P$. falciparum derived from etacrynic acid. Molecules 2008; 14(1):19-35. 
135. Oliveira R, Guedes RC, Meireles P, Albuquerque IS, Gonçalves LM, Pires E, et al. Tetraoxane-pyrimidine nitrile hybrids as dual stage anti-malarials. J Med Chem 2014; 57(11):4916-4923.

136. Weldon DJ, Shah F, Chittiboyina AG, Sheri A, Chada RR, Gut J, et al. Synthesis, biological evaluation, hydration site thermodynamics, and chemical reactivity analysis of $\alpha$-keto substituted peptidomimetics for the inhibition of Plasmodium falciparum. Bioorg Med Chem Lett 2014; 24(5):1274-1279.

137. Nizi E, Sferrazza A, Fabbrini D, Nardi V, Andreini M, Graziani $\mathrm{R}$, et al. Peptidomimetic nitrile inhibitors of malarial protease falcipain-2 with high selectivity against human cathepsins. Bioorg Med Chem Lett 2018; 28(9):1540-1544.

138. Marques AF, Esser D, Rosenthal PJ, Kassack MU, Lima LM. Falcipain-2 inhibition by suramin and suramin analogues. Bioorg Med Chem. 2013; 21(13):3667-3673.

139. Bertoldo JB, Chiaradia-Delatorre LD, Mascarello A, Leal PC, Cordeiro MN, Nunes RJ, et al. Synthetic compounds from an in house library as inhibitors of falcipain-2 from Plasmodium falciparum. J Enzyme Inhib Med Chem 2015; 30(2):299-307.

140.Pant A, Kumar R, Wani NA, Verma S, Sharma R, Pande $\mathrm{V}$, et al. Allosteric site inhibitor disrupting auto-processing of malarial cysteine proteases. Sci Rep 2018; 8(1):16193.

141. Boyom FF, Kemgne EM, Tepongning R, Ngouana $\mathrm{V}$, Mbacham WF, Tsamo E, et al. Anti-plasmodial activity of extracts from seven medicinal plants used in malaria treatment in Cameroon. J Ethnopharmacol 2009; 123(3):483-488.

142. Stolze SC, Deu E, Kaschani F, Li N, Florea BI, Richau $\mathrm{KH}$, et al. The antimalarial natural product symplostatin 4 is a nanomolar inhibitor of the food vacuole falcipains. Chem Biol 2012; 19(12):15461555.

143. Tadigoppula N, Korthikunta V, Gupta S, Kancharla P, Khaliq T, Soni A, et al. Synthesis and insight into the structure-activity relationships of chalcones as antimalarial agents. J Med Chem 2013; 56(1):3145.

144. Wang L, Zhang S, Zhu J, Zhu L, Liu X, Shan L, et al. Identification of diverse natural products as falcipain-2 inhibitors through structure-based virtual screening. Bioorg Med Chem Lett 2014; 24(5):1261-1264.

145. Musyoka TM, Kanzi AM, Lobb KA, Tastan Bishop Ö. Structure based docking and molecular dynamic studies of plasmodial cysteine proteases against a South African natural compound and its analogs. Sci Rep 2016; 6:23690.

146. Pereira GAN, Souza GC, Santos LS, Barata LES, Meneses CCF, Krettli AU, et al. Synthesis, antimalarial activity in vitro, and docking studies of novel neolignan derivatives. Chem Biol Drug Des 2017; 90(3):464-472.
147. Himangini, Pathak DP, Sharma V, Kumar S Designing novel inhibitors against falcipain-2 of Plasmodium falciparum. Bioorg Med Chem Lett 2018; 28(9):1566-1569.

148. Jin H, Xu Z, Cui K, Zhang T, Lu W, Huang J. Dietary flavonoids fisetin and myricetin: dual inhibitors of Plasmodium falciparum falcipain-2 and plasmepsin II. Fitoterapia 2014; 94:55-61.

149. Shibi IG, Aswathy L, Jisha RS, Masand VH, Gajbhiye JM. Virtual screening techniques to probe the antimalarial activity of some traditionally used phytochemicals. Comb Chem High Throughput Screen 2016; 19(7):572-591.

150. Melo PMS, El ChamyMaluf S, Azevedo MF, Paschoalin T, Budu A, Bagnaresi P, et al. Short communication: Inhibition of Plasmodium falciparum cysteine proteases by the sugarcane cystatin Cane CPI-4. Parasitol Int 2018; 67(2):233236.

151.Pandey KC, Singh N, Arastu-Kapur S, Bogyo M, Rosenthal PJ. Falstatin, a cysteine protease inhibitor of Plasmodium falciparum, facilitates erythrocyte invasion. PLoS Pathog 2006; 2(11):e117.

152. Rennenberg A, Lehmann C, Heitmann A, Witt T, Hansen G, Nagarajan $\mathrm{K}$, et al. Exoerythrocytic Plasmodium parasites secrete a cysteine protease inhibitor involved in sporozoite invasion and capable of blocking cell death of host hepatocytes. PLoS Pathog 2010; 6(3):e1000825.

153. Pei Y, Miller JL, Lindner SE, Vaughan AM, Torii M, Kappe SH. Plasmodium yoelii inhibitor of cysteine proteases is exported to exomembrane structures and interacts with yoelipain-2 during asexual blood-stage development. Cell. Microbiol 2013:15(9):1508-1526.

154. Boysen KE, Matuschewski K. Inhibitor of cysteine proteases is critical for motility and infectivity of Plasmodium sporozoites. MBio 2013; 4(6):e00874-13.

155. Lehmann C, Heitmann A, Mishra S, Burda PC, Singer M, Prado $\mathrm{M}$, et al. A cysteine protease inhibitor of Plasmodium berghei is essential for exo-erythrocytic development. PLoS Pathog 2014; 10(8):e1004336.

156. Hansen G, Schwarzloh B, Rennenberg A, Heussler VT, Hilgenfeld R. The macromolecular complex of ICP and falcipain-2 from Plasmodium: preparation, crystallization and preliminary X-ray diffraction analysis. Acta Crystallogr Sect F Struct Biol Cryst Commun 2011; 67(11): 1406-1410.

157. Hansen G, Heitmann A, Witt T, Li H, Jiang H, Shen $\mathrm{X}$, et al. Structural basis for the regulation of cysteine-protease activity by a new class of protease inhibitors in Plasmodium. Structure 2011; 19(7):919-9129.

158.Sundararaj S, Saxena AK, Sharma R, Vashisht K, Sharma S, Anvikar A, et al. Cross-talk between malarial cysteine proteases and falstatin: the BC loop as a hot-spot target. PLoS One 2014; 9(4):e93008. 